\title{
Surface plasticity: theory and computation
}

\author{
A. Esmaeili ${ }^{1}$ - P. Steinmann ${ }^{1} \cdot$ A. Javili ${ }^{2}$ \\ Received: 12 July 2017 / Accepted: 14 November 2017 / Published online: 22 November 2017 \\ (c) Springer-Verlag GmbH Germany, part of Springer Nature 2017
}

\begin{abstract}
Surfaces of solids behave differently from the bulk due to different atomic rearrangements and processes such as oxidation or aging. Such behavior can become markedly dominant at the nanoscale due to the large ratio of surface area to bulk volume. The surface elasticity theory (Gurtin and Murdoch in Arch Ration Mech Anal 57(4):291-323, 1975) has proven to be a powerful strategy to capture the size-dependent response of nano-materials. While the surface elasticity theory is well-established to date, surface plasticity still remains elusive and poorly understood. The objective of this contribution is to establish a thermodynamically consistent surface elastoplasticity theory for finite deformations. A phenomenological isotropic plasticity model for the surface is developed based on the postulated elastoplastic multiplicative decomposition of the surface superficial deformation gradient. The non-linear governing equations and the weak forms thereof are derived. The numerical implementation is carried out using the finite element method and the consistent elastoplastic tangent of the surface contribution is derived. Finally, a series of numerical examples provide further insight into the problem and elucidate the key features of the proposed theory.
\end{abstract}

Keywords Surface elasticity $\cdot$ Surface plasticity $\cdot$ Finite element method

\section{Introduction}

The boundary of a continuum body can display its own distinct properties compared to those of the bulk, e.g. due to broken atomic bonds at the surface. This phenomenon is usually modeled via surface stresses $[13,25,30,42,64]$ associated with a zero-thickness layer on the material. The surface stress can also be derived from a boundary potential when one deals with a conservative formulation. Such surface potential usually depends only on the surface deformation gradient and also on the surface normal in the case of anisotropy. In addition, the difference between the properties of the boundary of a continuum body and those of the bulk can also be

$凶 \quad$ A. Javili

ajavili@bilkent.edu.tr

A. Esmaeili

ali.esmaeili@1tm.uni-erlangen.de

P. Steinmann

paul.steinmann@1tm.uni-erlangen.de

1 Chair of Applied Mechanics, University of Erlangen-Nuremberg, Egerlandstrasse 5, 91058 Erlangen, Germany

2 Department of Mechanical Engineering, Bilkent University, 06800 Ankara, Turkey due to exposing the surface to processes such as oxidation, aging, grit blasting, plasma jet treatment, etc. Phenomenological modeling of such surfaces is achieved by endowing the surface with its own energy, and further surface specific thermodynamic ingredients, see $[1,27,36-39,41,64,65]$.

In the context of the current work, due to some confusion in the literature, the definitions of the terms surface stress and surface energy shall be re-iterated. Surface energy is usually understood as an excess energy term due to the presence of the surface as an enegetic layer or also as a superficial energy term due to rearrangement of atoms very close to a surface $[25,40]$. Alternatively, one can regard the surface energy to be associated with either the creation of a new surface at constant strain or the deformation (straining) of the already existing surface [43], see also [23,57,58,67]. Surface stress is the force responsible for elastically deforming the surface of the body resulting in the change of the distances among atoms or molecules on the surface [42].

In the current work, we build upon the surface elasticity theory of Gurtin and Murdoch [30]. The surface elasticity theory is a well-established methodology to capture the sizedependent behavior of materials at the nano-scale and has been extensively studied in the past decades, see e.g. [5,7$9,12,28,31,36,44-47,50,56,63,66,69]$ and references therein. 
The effect of surface energetics, e.g. for inclusions, and the size-dependent elastic state of the material has recently been investigated for instance in $[3,4,6,10,13-17,24,33,49,53-$ $55,68]$ and references therein. The numerical simulation of surfaces has been realized in $[2,48,51,52]$ when the bulk behaves like a fluid, and in [34-38] for solids.

Nonetheless, the surface elasticity theory suffers from the fact that the surface behavior remains elastic regardless of the strain level at the surface. To address this problem, the authors have recently extended the surface elasticity theory to also account for damage along the surface [18-22]. The objective of this contribution is to further extend surface elasticity to account for another form of surface inelasticity, i.e. plasticity along the surface. Although the plasticity of interfaces specifically in the context of grain boundaries and gradient plasticity formulations has been considered by various authors [26,29], to the best of the authors' knowledge in $[26,29]$ the bulk formulation therein is a gradient plasticity model with corresponding consequences for the attached surface. The resulting modeling of inelastic interfaces is thus indeed different from the one pursued in the present work. Here surface plasticity is based on the concept of a surface (superficial) deformation gradient and thus corresponds conceptually to plasticity of a thin layer of material at the microscale that can be modeled by an effective twodimensional surface attached to an ordinary first-order bulk. The surface elasticity theory of Gurtin and Murdoch [30] has been one of the most cited papers in the past decade mainly due to emerging applications of nano-materials and the utility of the surface elasticity theory to predict the material behavior at the nano-scale where the surface to volume ratio increases dramatically. Likewise, the surface plasticity theory here aims to provide a generic framework suitable for understanding plastic-like material behavior at small scales where the surface effects are no longer negligible. From a geometrical viewpoint, both surface and membrane are two-dimensional manifolds in a three dimensional Euclidean space and thus identical. However, from a material viewpoint, a membrane can exist by itself and without a bulk, unlike a surface. Surface is always the boundary of a bulk and cannot be defined without an encased bulk. This subtle difference between the surface and membrane leads to various surprising outcomes. For instance, the surface Young's modulus $\hat{E}$ is not required to be positive, whereas for a membrane, the positive definiteness requires the Young's modulus to be positive. Such distinctions between surface elasticity theory and membrane theory stem from the "kinematic slavery condition" of the surface which does not hold for the membrane.

The surface plasticity theory here is the natural extension of the surface elasticity theory of Gurtin and Murdoch [30] capable to capture size-effects, unlike the first-order continuum mechanics. The proposed theory and the implementation aspects are very general and can be applied to various scenarios. Note, the material modeling of the bulk is a mature field with many standard references and associated experiments. This is not the case for the surface though. There have been several theoretical studies on surface elasticity, but there are very few experiments for measuring the materials constants. Nevertheless, without a clear theoretical framework no experimental evidence can be obtained. We believe that sooner or later new surface plasticity coefficients will be measured and the relationship between the propagation of dislocations in the bulk and that on the surface becomes more clear. The same argument holds for the surface hardening. Only equipped with a generic surface plasticity theory, one can measure surface hardening and explains its nature.

The main contributions of this work is the extension of surface elasticity into a phenomenological isotropic ${ }^{1}$ surface plasticity model based on the notion of an intermediate stressfree configuration. Thereby the phenomenological plasticity model on the surface proposed here rests on the multiplicative decomposition of the superficial surface deformation gradient $\hat{\boldsymbol{F}}$ (independent from the corresponding multiplicative decomposition in the bulk). Subsequently, for the sake of demonstration, a model problem that includes the simplest surface plasticity formulation, i.e. $\mathrm{J}_{2}$ type surface flow theory with isotropic hardening is developed and used for the numerical examples to also study the computational aspects of surface elastoplasticity. In doing so, we compare the mechanical response of the computational domain under various circumstances where the bulk and/or the surface are allowed to respond plastically. The plasticity in the bulk closely follows the works of Simo et al. [59,60]. For the sake of brevity, we exclude the details of the elastoplastic bulk formulation and refer the interested reader to $[11,32,61]$. In summary, the key contributions of this work are as follows:

- To review the governing equations of a body possessing an energetic surface in a finite-deformation setting, extend them to include plasticity on the surface and to derive the weak form of the local balance of forces on the surface.

- To present a thermodynamically consistent formulation resorting to the dissipation inequality on the surface.

- To derive the consistent tangent stiffness matrix on the surface.

- To illustrate the theory with the help of numerical examples using the finite element method.

The manuscript is organized as follows. Section 2 summarizes the kinematics of non-linear continuum mechanics including elastoplastic surfaces. The governing equations in the bulk and on the surface including the balance equations and the surface dissipation inequality are given in Sect. 3 .

\footnotetext{
1 The assumption of isotropy in this work is for the sake of simplicity.
} 
A general surface plastic yield condition and the evolution equations are derived in Sect. 3.1. A specific surface yield criterion and the kinematics of a surface volumetric-deviatoric decomposition are discussed in Sect. 3.2. The decoupled hyperelastic part of the model, the return mapping algorithm and the exact linearization of the elastoplastic surface update formula are presented in Sect. 3.3. A numerical framework that encompasses surface elastoplasticity is established in Sect. 4. The framework includes the weak formulation of the governing equations and the corresponding finite element implementation. A series of numerical examples based on the finite element approximation of the weak form is presented in Sect. 5 to elucidate the theory. Section 6 concludes this work.

\section{Kinematics}

This section summarizes the kinematics of non-linear continuum mechanics including elastoplastic surfaces and introduces the notation adopted here.

Consider a continuum body $\mathscr{B}$ that takes the material configuration $\mathcal{B}_{0} \subset \mathbb{E}^{3}$ at time $t=0$, and the spatial configuration $\mathcal{B}_{t}$ at $t>0$, as depicted in Fig. 1. The bulk is defined by $\mathcal{B}_{0}$, with reference (material) and current (spatial) placements of material particles labeled $\boldsymbol{X}$ and $\boldsymbol{x}$, respectively. The boundary of the bulk is described by a lower-dimensional manifold (surface) embedded in the threedimensional Euclidean space and is denoted by $\mathcal{S}_{0}=\partial \mathcal{B}_{0}$ and $\mathcal{S}_{t}=\partial \mathcal{B}_{t}$. The boundary placements in the material and spatial configurations are defined by $\hat{\boldsymbol{X}}$ and $\hat{\boldsymbol{x}}$, respectively. All hatted quantities $\{\hat{\boldsymbol{\bullet}}\}$ refer to the surface. The outward unit normal to $\partial \mathcal{B}_{0}$ and $\partial \mathcal{B}_{t}$ are denoted respectively by $N$ and $\boldsymbol{n}$. The deformation maps of the bulk and the encompassing surface are denoted by $\varphi$ and $\hat{\varphi}$, respectively. Thus $\boldsymbol{x}=\boldsymbol{\varphi}(\boldsymbol{X}, t)$ and $\hat{\boldsymbol{x}}=\hat{\boldsymbol{\varphi}}(\hat{\boldsymbol{X}}, t)$. The inverse deformation maps of the bulk and the surface are denoted by $X=\varphi^{-1}(x, t)$ and $\hat{\boldsymbol{X}}=\hat{\boldsymbol{\varphi}}^{-1}(\hat{\boldsymbol{x}}, t)$, respectively. The bulk and the (rankdeficient) surface deformation gradients $\boldsymbol{F}$ and $\hat{\boldsymbol{F}}$, together with the corresponding velocities $\boldsymbol{V}$ and $\hat{\boldsymbol{V}}$ are, respectively, defined by

$$
\begin{array}{ll}
\boldsymbol{F}(\boldsymbol{X}, t):=\operatorname{Grad} \boldsymbol{\varphi}(\boldsymbol{X}, t), \quad \boldsymbol{V}:=\mathrm{D}_{t} \boldsymbol{\varphi}(\boldsymbol{X}, t) \quad \text { and } \\
\hat{\boldsymbol{F}}(\hat{\boldsymbol{X}}, t):=\widehat{\operatorname{Grad}} \hat{\boldsymbol{\varphi}}(\hat{\boldsymbol{X}}, t), \quad \hat{\boldsymbol{V}}:=\mathrm{D}_{t} \hat{\boldsymbol{\varphi}}(\hat{\boldsymbol{X}}, t) .
\end{array}
$$

Thereby the surface gradient and divergence operators, respectively, read

$$
\begin{aligned}
& \widehat{\operatorname{Grad}}\{\hat{\bullet}\}:=\operatorname{Grad}\{\hat{\bullet}\} \cdot \hat{\boldsymbol{I}} \text { and } \\
& \widehat{\operatorname{Div}}\{\hat{\bullet}\}:=\widehat{\operatorname{Grad}}\{\hat{\bullet}\}: \hat{\boldsymbol{I}} \quad \text { with } \hat{\boldsymbol{I}}:=\boldsymbol{I}-\hat{\boldsymbol{N}} \otimes \hat{\boldsymbol{N}},
\end{aligned}
$$

where $\hat{\boldsymbol{I}}$ and $\boldsymbol{I}$ denote the surface and bulk unit tensors. Their spatial counterparts are denoted $\hat{\boldsymbol{i}}$ and $\boldsymbol{i}$. Moreover, the surface unit tensors can also be defined using the surface deformation gradient and the definition of its inverse as follows:

$$
\hat{\boldsymbol{F}} \cdot \hat{\boldsymbol{F}}^{-1}=\hat{\boldsymbol{i}} \text { and } \hat{\boldsymbol{F}}^{-1} \cdot \hat{\boldsymbol{F}}=\hat{\boldsymbol{I}} .
$$

Note that surface deformation gradient $\hat{\boldsymbol{F}}$ is not invertible. For further details on how the definitions of the inverse must be computed see [19]. Finally the bulk and surface Jacobians are denoted by $J:=\operatorname{det} \boldsymbol{F}>0$, and $\hat{J}:=\hat{\operatorname{det}} \hat{\boldsymbol{F}}>0$, respectively, with $\hat{\operatorname{det}}\{\bullet\}$ denoting the area determinant [64].

The underlying hypothesis of the proposed finite deformation surface elastoplasticity is the assumption of a multiplicative decomposition of the surface deformation gradient $\hat{\boldsymbol{F}}$ into an elastic $\hat{\boldsymbol{F}}_{\mathrm{e}}$ and a plastic surface distortion $\hat{\boldsymbol{F}}_{\mathrm{p}}$ :

$\hat{\boldsymbol{F}}=\hat{\boldsymbol{F}}_{\mathrm{e}} \cdot \hat{\boldsymbol{F}}_{\mathrm{p}}$.

This decomposition is based on the idea of a so-called intermediate surface stress-free configuration. This configuration can be obtained either starting from the material configuration through the application of $\hat{\boldsymbol{F}}_{\mathrm{p}}$ or starting from the current configuration by a pure elastic and local unloading through $\hat{\boldsymbol{F}}_{\mathrm{c}}^{-1}$. In other words the inverse of the surface elastic deformation gradient $\hat{\boldsymbol{F}}_{\mathrm{e}}^{-1}$ releases elastically the surface stress in the neighborhood of a surface point in the current configuration. See Fig. 1 for a depiction of the multiplicative decomposition of the surface deformation gradient and the corresponding configurations.

Remark 1 Micromechanically, in two-dimensional crystals the plastic surface distortion $\hat{\boldsymbol{F}}_{\mathrm{p}}$ is responsible for the microscopic glide of dislocation through the crystalline lattice, whereas the elastic surface distortion $\hat{\boldsymbol{F}}_{\mathrm{e}}$ is a measure of distortion and rotation of the lattice.

Remark 2 The elastic and plastic surface distortions, $\hat{\boldsymbol{F}}_{\mathrm{e}}$ and $\hat{\boldsymbol{F}}_{\mathrm{p}}$, respectively, do not necessarily depend on the corresponding bulk distortions in a similar way as the total surface deformation gradient $\hat{\boldsymbol{F}}$ depends on the total bulk deformation gradient $\boldsymbol{F}$, i.e. $\hat{\boldsymbol{F}}=\boldsymbol{F} \cdot \hat{\boldsymbol{I}}$. We imagine a thin layer of surface material that can have its own elastic and plastic decomposition of the deformation gradient $\hat{\boldsymbol{F}}$ independently of the bulk. One could imagine for instance an extreme case with a purely elastic bulk, $\boldsymbol{F}=\boldsymbol{F}_{\mathrm{e}}$, combined with an elastoplastic surface where $\hat{\boldsymbol{F}}=\boldsymbol{F}_{\mathrm{e}} \cdot \hat{\boldsymbol{I}}$ with $\hat{\boldsymbol{F}}=\hat{\boldsymbol{F}}_{\mathrm{e}} \cdot \hat{\boldsymbol{F}}_{\mathrm{p}}$.

For the subsequent developments of surface elastoplasticity we consider the following surface strain measures:

$\hat{\boldsymbol{C}}_{\mathrm{p}}:=\hat{\boldsymbol{F}}_{\mathrm{p}}^{\mathrm{t}} \cdot \hat{\boldsymbol{F}}_{\mathrm{p}}$ and $\hat{\boldsymbol{b}}_{\mathrm{e}}:=\hat{\boldsymbol{F}}_{\mathrm{e}} \cdot \hat{\boldsymbol{F}}_{\mathrm{e}}^{\mathrm{t}}$ where

$\hat{\boldsymbol{C}}_{\mathrm{p}}:=\hat{\boldsymbol{F}}^{\mathrm{t}} \cdot \hat{\boldsymbol{b}}_{\mathrm{e}}^{-1} \cdot \hat{\boldsymbol{F}}$. 


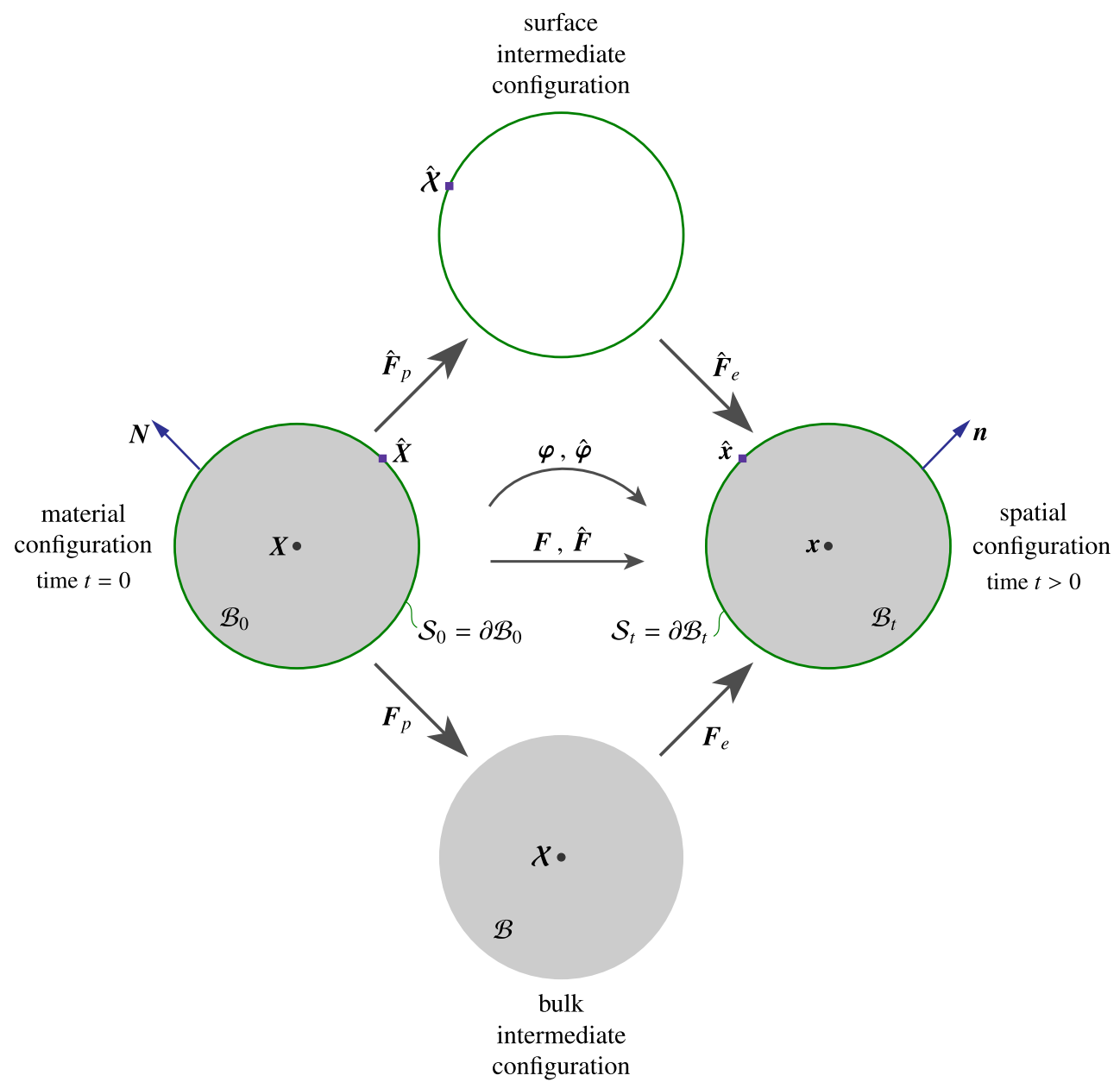

Fig. 1 The bulk domain $\mathcal{B}_{0}$, the surface $\mathcal{S}_{0}$, and the unit normals to the surface $N$, all defined in the material configuration. The bulk, surface deformation maps, denoted as $\varphi, \hat{\varphi}$, respectively, map the material configuration to the spatial configuration at time $t$. The bulk domain $\mathcal{B}_{t}$, the surface $\mathcal{S}_{t}$ and the unit normal to the surface $\boldsymbol{n}$, all defined in the spatial configuration. The bulk and (rank-deficient) surface total deformation gradients are $\boldsymbol{F}$ and $\hat{\boldsymbol{F}}$, respectively. Micromechanically the material is distorted by $\boldsymbol{F}_{\mathrm{p}}$ and $\hat{\boldsymbol{F}}_{\mathrm{p}}$ into the fictitious intermediate configuration by

Note that from a geometric point of view the surface right Cauchy-Green tensor $\hat{\boldsymbol{C}}$ and and its plastic counterpart $\hat{\boldsymbol{C}}_{\mathrm{p}}$ are the pull-backs of $\hat{\boldsymbol{i}}$ and $\hat{\boldsymbol{b}}_{\mathrm{e}}^{-1}$ where $\hat{\boldsymbol{i}}$ is the surface Euclidean metric in the current configuration and $\hat{\boldsymbol{b}}_{\mathrm{e}}^{-1}$ is the inverse of the surface elastic left Cauchy-Green tensor $\hat{\boldsymbol{b}}_{\mathrm{e}}$. The determinants of the elastic and plastic surface distortions are defined by $\hat{J}_{\mathrm{e}}:=\operatorname{det} \hat{\boldsymbol{F}}_{\mathrm{e}}>0$ and $\hat{J}_{\mathrm{p}}:=\operatorname{det} \hat{\boldsymbol{F}}_{\mathrm{p}}>0$, so that $\hat{J}=\hat{J}_{\mathrm{p}} \hat{J}_{\mathrm{e}}$.

Next, the spatial surface gradient of the spatial surface velocity $\hat{\boldsymbol{v}}(\hat{\boldsymbol{x}}, t)$ reads

$\widehat{\operatorname{grad}} \hat{\boldsymbol{v}}(\hat{\boldsymbol{x}}, t)=\hat{\boldsymbol{l}}=\dot{\hat{\boldsymbol{F}}} \cdot \hat{\boldsymbol{F}}^{-1}$

Noting the elastic-plastic decomposition of the surface deformation gradient, Eq. (6) is written as dislocation motion. The multiplicative decomposition takes the form $\boldsymbol{F}=\boldsymbol{F}_{\mathrm{e}} \cdot \boldsymbol{F}_{\mathrm{p}}$ and $\hat{\boldsymbol{F}}=\hat{\boldsymbol{F}}_{\mathrm{e}} \cdot \hat{\boldsymbol{F}}_{\mathrm{p}}$ in the bulk and independently on the surface. The elastic and plastic surface distortions, $\hat{\boldsymbol{F}}_{\mathrm{e}}$ and $\hat{\boldsymbol{F}}_{\mathrm{p}}$, respectively, do not necessarily depend on the corresponding bulk distortions in a similar way as the total surface deformation gradient $\hat{\boldsymbol{F}}$ depends on the total bulk deformation gradient $\boldsymbol{F}$, i.e. $\hat{\boldsymbol{F}}=\boldsymbol{F} \cdot \hat{\boldsymbol{I}}$. The elastic contributions to the total deformations rotate and distort the bulk and the surface. Both $\hat{\boldsymbol{F}}_{\mathrm{p}}$ and $\hat{\boldsymbol{F}}_{\mathrm{e}}$ are in general rank-deficient

$\hat{\boldsymbol{l}}=\hat{\boldsymbol{l}}_{\mathrm{e}}+\hat{\boldsymbol{F}}_{\mathrm{e}} \cdot \hat{\boldsymbol{L}}_{\mathrm{p}} \cdot \hat{\boldsymbol{F}}_{e}^{-1}$ where $\hat{\boldsymbol{l}}_{\mathrm{e}}=\dot{\hat{\boldsymbol{F}}}_{\mathrm{e}} \cdot \hat{\boldsymbol{F}}_{\mathrm{e}}^{-1}$ and

$\hat{\boldsymbol{L}}_{\mathrm{p}}=\dot{\hat{\boldsymbol{F}}}_{\mathrm{p}} \cdot \hat{\boldsymbol{F}}_{\mathrm{p}}^{-1}$.

with $\hat{\boldsymbol{l}}_{\mathrm{e}}$ and $\hat{\boldsymbol{L}}_{\mathrm{p}}$ being elastic and plastic surface "velocity gradients". Although the surface velocity gradient $\hat{l}$ itself and the elastic contribution $\hat{l}_{\text {e }}$ are spatial quantities, the surface plastic velocity gradient $\hat{\boldsymbol{L}}_{\mathrm{p}}$ is associated with the intermediate configuration which is why in Eq. (7) 1 a push-forward to the current configuration is applied. Finally the surface Lie derivative $^{2}$ of Eq. $(5)_{2}$ reads

2 The Lie derivative of a spatial surface tensor field $\hat{\boldsymbol{f}}(\hat{\boldsymbol{x}}, t)$, relative to a vector field $\hat{\boldsymbol{v}}$ is obtained by $£_{\hat{v}} \hat{\boldsymbol{f}}=\hat{\boldsymbol{\varphi}}_{*}\left(\frac{\mathrm{D}}{\mathrm{D} t} \hat{\boldsymbol{\varphi}}_{*}^{-1}(\hat{\boldsymbol{f}})\right)$, where $\hat{\boldsymbol{\varphi}}_{*}^{-1}$ is the surface pull-back and $\hat{\varphi}_{*}$ the surface push-forward operators. 
Table 1 Localized force and moment balances in the bulk and on the surface in the material configuration

\begin{tabular}{|c|c|c|c|}
\hline & \multirow[t]{2}{*}{ Force balance } & $\operatorname{Div} \boldsymbol{P}+\boldsymbol{B}^{\mathrm{p}}=\mathbf{0}$ & in $\mathcal{B}_{0}$ \\
\hline & & $\widehat{\operatorname{Div}} \hat{\boldsymbol{P}}+\hat{\boldsymbol{B}}^{\mathrm{p}}-\boldsymbol{P} \cdot \boldsymbol{N}=\mathbf{0}$ & on $\mathcal{S}_{0}$ \\
\hline & \multirow[t]{2}{*}{ Moment balance ${ }^{\mathrm{a}}$} & $\boldsymbol{P} \cdot \boldsymbol{F}^{t}=\boldsymbol{F} \cdot \boldsymbol{P}^{t}$ & in $\mathcal{B}_{0}$ \\
\hline & & $\hat{\boldsymbol{P}} \cdot \hat{\boldsymbol{F}}^{t}=\hat{\boldsymbol{F}} \cdot \hat{\boldsymbol{P}}^{t}$ & on $\mathcal{S}_{0}$ \\
\hline $\boldsymbol{B}^{\mathrm{p}}$ & Force vector per unit volume & $\hat{\boldsymbol{B}}^{\mathrm{p}}$ & Surface traction per unit area \\
\hline
\end{tabular}

The notation $\{\bullet\}^{\mathrm{p}}$ is to denote prescribed quantities

${ }^{a}$ Balance of angular momentum results in the symmetry of the bulk and surface Cauchy stress $£_{\hat{\boldsymbol{v}}} \hat{\boldsymbol{b}}_{\mathrm{e}}=\dot{\hat{\boldsymbol{b}}}_{\mathrm{e}}-\hat{\boldsymbol{l}} \cdot \hat{\boldsymbol{b}}_{\mathrm{e}}-\hat{\boldsymbol{b}}_{\mathrm{e}} \cdot \hat{\boldsymbol{l}}^{\mathrm{t}}$ with $£_{\hat{v}} \hat{\boldsymbol{b}}_{\mathrm{e}}=\hat{\boldsymbol{F}} \cdot \dot{\hat{\boldsymbol{C}}}_{\mathrm{p}}^{-1} \cdot \hat{\boldsymbol{F}}^{\mathrm{t}}$.

\section{Governing equations}

The local balance equations of forces and moments in the bulk and on the surface are listed in Table 1.

Restricting the material response to isotropy both on the surface and in the bulk, the arguments ${ }^{3}$ of the corresponding free energies are chosen as

$\Psi \equiv \Psi\left(\boldsymbol{b}_{\mathrm{c}}, \boldsymbol{\alpha}\right) \quad$ and $\quad \hat{\Psi} \equiv \Psi\left(\hat{\boldsymbol{b}}_{\mathrm{e}}, \hat{\boldsymbol{\alpha}}\right)$

where $\boldsymbol{\alpha}$ and $\hat{\boldsymbol{\alpha}}$ are the internal variables characterizing the state of bulk and surface strain hardening, respectively. Next the reduced dissipation inequality on the surface is exploited. By differentiating Eq. (9) 2 with respect to time, using Eq. (7), and the isotropy assumption, renders

$$
\begin{aligned}
\dot{\hat{\Psi}}\left(\hat{\boldsymbol{b}}_{\mathrm{e}}, \hat{\boldsymbol{\alpha}}\right)= & \frac{\partial \hat{\Psi}}{\partial \hat{\boldsymbol{b}}_{\mathrm{e}}}: \dot{\hat{\boldsymbol{b}}}_{\mathrm{e}}+\frac{\partial \hat{\Psi}}{\partial \hat{\boldsymbol{\alpha}}} \circ \dot{\hat{\boldsymbol{\alpha}}} \\
= & {\left[\frac{\partial \hat{\Psi}}{\partial \hat{\boldsymbol{b}}_{\mathrm{e}}} \cdot \hat{\boldsymbol{b}}_{\mathrm{e}}\right]:\left[2 \hat{\boldsymbol{l}}+£_{\hat{\boldsymbol{v}}} \hat{\boldsymbol{b}}_{\mathrm{e}} \cdot \hat{\boldsymbol{b}}_{\mathrm{e}}^{-1}\right] } \\
& +\frac{\partial \hat{\Psi}}{\partial \hat{\boldsymbol{\alpha}}} \circ \dot{\hat{\boldsymbol{\alpha}}}
\end{aligned}
$$

where $\circ$ is a general contraction operator whose order of contraction depends on weather $\hat{\boldsymbol{\alpha}}$ is scalar or tensorial. Particularizing next the surface Clausius-Plank inequality and

\footnotetext{
${ }^{3}$ Note that the most general set of arguments for the surface free energy contains $\hat{\boldsymbol{F}}_{\mathrm{p}}$ and $\hat{\boldsymbol{F}}$ in Euclidean space. Imposing the invariance under superposed rigid body motion onto the intermediate configuration results in $\hat{\boldsymbol{C}}_{\mathrm{e}}$, where $\hat{\boldsymbol{C}}_{\mathrm{e}}$ is the elastic right Cauchy-Green strain in the intermediate configuration. Imposing further invariance under superposed rigid body motion on the spatial configuration finally results in $\hat{\boldsymbol{b}}_{\mathrm{e}}$, see [59] for further details.
}

using the relation $\hat{\boldsymbol{d}}=\hat{\boldsymbol{l}}^{\mathrm{sym}_{4}}$ one expresses the surface dissipation inequality $\hat{\mathcal{D}}$ as

$$
\begin{aligned}
\hat{\mathcal{D}}= & \hat{\boldsymbol{\tau}}: \hat{\boldsymbol{d}}-\dot{\hat{\Psi}} \geq 0 \Longrightarrow \hat{\mathcal{D}}=\left[\hat{\boldsymbol{\tau}}-2 \frac{\partial \hat{\Psi}}{\partial \hat{\boldsymbol{b}}_{\mathrm{e}}} \cdot \hat{\boldsymbol{b}}_{\mathrm{e}}\right]: \hat{\boldsymbol{d}} \\
& +\left[2 \frac{\partial \hat{\Psi}}{\partial \hat{\boldsymbol{b}}_{\mathrm{e}}} \cdot \hat{\boldsymbol{b}}_{\mathrm{e}}\right]:\left[-\frac{1}{2} £_{\hat{\boldsymbol{v}}} \hat{\boldsymbol{b}}_{\mathrm{e}} \cdot \hat{\boldsymbol{b}}_{\mathrm{e}}^{-1}\right]-\frac{\partial \hat{\Psi}}{\partial \hat{\boldsymbol{\alpha}}} \circ \dot{\hat{\boldsymbol{\alpha}}} \geq 0
\end{aligned}
$$

where the surface Kirchhoff stress $\hat{\boldsymbol{\tau}}$ is the push-forward of the surface Piola-Kirchhoff stress $\hat{\boldsymbol{S}}$. Thereby the following relations hold

$\hat{\boldsymbol{\tau}}=\hat{\boldsymbol{F}} \cdot \hat{\boldsymbol{S}} \cdot \hat{\boldsymbol{F}}^{\mathrm{t}}$ and $\hat{\boldsymbol{S}}=\hat{\boldsymbol{F}}^{-1} \cdot \hat{\boldsymbol{P}}$ and $\hat{\boldsymbol{\tau}}=\hat{\boldsymbol{P}} \cdot \hat{\boldsymbol{F}}^{\mathrm{t}}$

Following the Coleman-Noll exploitation we eventually find that

$\hat{\boldsymbol{\tau}}=2 \frac{\partial \hat{\Psi}}{\partial \hat{\boldsymbol{b}}_{\mathrm{e}}} \cdot \hat{\boldsymbol{b}}_{\mathrm{e}}$ and

$\hat{\mathcal{D}}_{\text {red }}=\hat{\boldsymbol{\tau}}:\left[-\frac{1}{2} £_{\hat{v}} \hat{\boldsymbol{b}}_{\mathrm{e}} \cdot \hat{\boldsymbol{b}}_{\mathrm{e}}^{-1}\right]-\frac{\partial \hat{\Psi}}{\partial \hat{\boldsymbol{\alpha}}} \circ \dot{\hat{\boldsymbol{\alpha}}} \geq 0$,

where Eq. $(13)_{1}$ is the surface constitutive relation and $\hat{\mathcal{D}}_{\text {red }}$ is the reduced dissipation inequality on the surface.

\subsection{Yield condition, maximum dissipation and evolution equations}

We now consider a surface yield function defined in the surface stress space. Let $\hat{\phi}(\hat{\boldsymbol{\tau}}, \hat{\boldsymbol{\beta}})$ be a general surface yield function dependent on the surface Kirchhoff stress and the stress-like surface internal variables (or conjugate thermodynamical forces to $\hat{\boldsymbol{\alpha}}$ ) denoted by $\hat{\boldsymbol{\beta}}=\partial \hat{\Psi} / \partial \hat{\boldsymbol{\alpha}}$. Let now $\hat{\mathscr{E}}$, $\partial \hat{\mathscr{E}}$ and $\hat{\mathscr{E}}$ be defined as

\footnotetext{
${ }^{4}$ The spatial symmetry operator is $\{\hat{\bullet}\}^{\text {sym }}={ }_{0}^{\text {sym }}:\{\hat{\bullet}\}$, where ${ }_{0}^{\text {sym }}=$ $\frac{1}{2}[\hat{\boldsymbol{i}} \overline{\boldsymbol{i}} \hat{\boldsymbol{i}}+\hat{\boldsymbol{i}} \otimes \hat{\boldsymbol{i}}]$, for spatial second-order surface tensors. Its Material counterpart is defined as $\{\hat{\boldsymbol{\theta}}\}^{\mathrm{Sym}}=\mathbb{I}^{\mathrm{Sym}}:\{\hat{\boldsymbol{\bullet}}\}$, where $\mathbb{I}^{\mathrm{Sym}}=\frac{1}{2}[\hat{\boldsymbol{I}} \bar{\otimes} \hat{\boldsymbol{I}}+$ $\hat{\boldsymbol{I}} \otimes \hat{\boldsymbol{I}}]$.
} 
$\hat{\mathscr{E}}=\{\hat{\boldsymbol{\tau}} \mid \hat{\phi}(\hat{\boldsymbol{\tau}}, \hat{\boldsymbol{\beta}})<0\}, \quad \partial \hat{\mathscr{E}}=\{\hat{\boldsymbol{\tau}} \mid \hat{\phi}(\hat{\boldsymbol{\tau}}, \hat{\boldsymbol{\beta}})=0\} \quad$ and

$\hat{\mathscr{E}}=\{\hat{\boldsymbol{\tau}} \mid \hat{\phi}(\hat{\boldsymbol{\tau}}, \hat{\boldsymbol{\beta}}) \leq 0\}$,

which are respectively the surface elastic domain, the surface yield surface (the boundary of $\hat{\mathscr{E}}$ ) and the surface admissible domain. Having defined the reduced dissipation inequality and the yield function on the surface, we state the principle of maximum plastic surface dissipation, which is used in associative plasticity to derive the flow rule and loading/unloading conditions in Kuhn-Tucker form. Locally, for a prescribed $\hat{\boldsymbol{b}}_{\mathrm{e}}$ and prescribed rates $\dot{\hat{\boldsymbol{C}}}_{\mathrm{p}}$ and $\dot{\hat{\boldsymbol{\alpha}}}$ (so that $£_{\hat{\boldsymbol{v}}} \hat{\boldsymbol{b}}_{\mathrm{e}}$ is fixed), among all possible surface stresses $\hat{\boldsymbol{\tau}}^{*}$ and stress-like internal variables $\hat{\boldsymbol{\beta}}^{*}$ satisfying Eq. (14) 3 , the plastic dissipation Eq. $(13)_{2}$ is maximized for the actual state $\left(\hat{\boldsymbol{\tau}}^{*}, \hat{\boldsymbol{\beta}}^{*}\right)$, i.e.

$\operatorname{Max}\left(\mathcal{D}_{\text {red }}\left(\hat{\boldsymbol{\tau}}^{*}, \hat{\boldsymbol{\beta}}^{*}, £_{\hat{\boldsymbol{v}}} \hat{\boldsymbol{b}}_{\mathrm{e}}, \dot{\hat{\boldsymbol{\alpha}}}\right)\right) \quad$ subject to the constraint

$\hat{\phi}\left(\hat{\boldsymbol{\tau}}^{*}, \hat{\boldsymbol{\beta}}^{*}\right) \leq 0$.

Equivalently, Eq. (15) 1 can be written as

$\left[\hat{\boldsymbol{\tau}}-\hat{\boldsymbol{\tau}}^{*}\right]:\left[-\frac{1}{2}\left[£_{\hat{\boldsymbol{v}}} \hat{\boldsymbol{b}}_{\mathrm{e}}\right] \cdot \hat{\boldsymbol{b}}_{\mathrm{e}}^{-1}\right]-\left[\hat{\boldsymbol{\beta}}-\hat{\boldsymbol{\beta}}^{*}\right] \circ \dot{\hat{\boldsymbol{\alpha}}} \geq 0$.

Now the flow rule and loading/unloading conditions can be obtained as follows: first we transform the inequality Eq. (13) 2 into a minimization problem. Next the constrained minimization problem is reformulated into an unconstrained problem by introducing the Lagrange multiplier $\gamma \geq 0$. Thus the Lagrangian functional $\hat{\mathcal{L}}$ reads

$\hat{\mathcal{L}}:=-\mathcal{D}_{\text {red }}\left(\hat{\boldsymbol{\tau}}^{*}, \hat{\boldsymbol{\beta}}^{*}, £_{\hat{\boldsymbol{v}}} \hat{\boldsymbol{b}}_{\mathrm{e}}, \dot{\hat{\boldsymbol{\alpha}}}\right)+\gamma \hat{\phi}\left(\hat{\boldsymbol{\tau}}^{*}, \hat{\boldsymbol{\beta}}^{*}\right)$.

For the stationary points of $\hat{\mathcal{L}}$, the derivatives $\partial \hat{\mathcal{L}} / \partial \hat{\boldsymbol{\tau}}, \partial \hat{\mathcal{L}} / \partial \hat{\boldsymbol{\beta}}$ and $\partial \hat{\mathcal{L}} / \partial \gamma$ must vanish, thus

$-\frac{1}{2} £_{\hat{v}} \hat{\boldsymbol{b}}_{\mathrm{e}}=\gamma \frac{\partial \hat{\phi}}{\partial \hat{\boldsymbol{\tau}}} \cdot \hat{\boldsymbol{b}}_{\mathrm{e}}, \quad \dot{\hat{\boldsymbol{\alpha}}}=\gamma \frac{\partial \hat{\phi}}{\partial \hat{\boldsymbol{\beta}}}$

$\gamma \geq 0, \quad \hat{\phi}(\hat{\boldsymbol{\tau}}, \hat{\boldsymbol{\beta}}) \leq 0, \quad \gamma \hat{\phi}(\hat{\boldsymbol{\tau}}, \hat{\boldsymbol{\beta}})=0$.

Remark 3 For isotropy the flow rule Eq. (18) $)_{1}$ is equivalent to

$\gamma \partial \hat{\phi} / \partial \hat{\boldsymbol{\tau}}=\hat{\boldsymbol{F}}_{\mathrm{e}} \cdot \hat{\boldsymbol{L}}_{\mathrm{p}} \cdot \hat{\boldsymbol{F}}_{\mathrm{e}}^{-1}$,

which may be shown as follows ${ }^{5}$ : by multiplying both sides of the above relation by $\hat{\boldsymbol{b}}_{\mathrm{e}}$, noting $\hat{\boldsymbol{F}}_{\mathrm{e}} \cdot \hat{\boldsymbol{L}}_{\mathrm{p}} \cdot \hat{\boldsymbol{F}}_{\mathrm{e}}^{-1}=\hat{\boldsymbol{l}}-\hat{\boldsymbol{l}}_{\mathrm{e}}$ and that the left hand side in Eq. (19) is symmetric (due to the

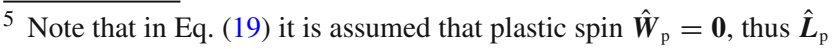
is symmetric.
}

isotropy assumption the tensor $\hat{\boldsymbol{b}}_{\mathrm{e}}$ and $\partial \hat{\phi} / \partial \hat{\boldsymbol{\tau}}$ commute), we obtain

$\gamma \frac{\partial \hat{\phi}}{\partial \hat{\boldsymbol{\tau}}} \cdot \hat{\boldsymbol{b}}_{\mathrm{e}}=\frac{1}{2}\left[\hat{\boldsymbol{l}} \cdot \hat{\boldsymbol{b}}_{\mathrm{e}}+\hat{\boldsymbol{b}}_{\mathrm{e}} \cdot \hat{\boldsymbol{l}}^{\mathrm{t}}-\dot{\hat{\boldsymbol{b}}}_{\mathrm{e}}\right]$ with

$\dot{\hat{\boldsymbol{b}}}_{\mathrm{e}}=\dot{\hat{\boldsymbol{F}}}_{\mathrm{e}} \cdot \hat{\boldsymbol{F}}_{\mathrm{e}}^{\mathrm{t}}+\hat{\boldsymbol{F}}_{\mathrm{e}}^{\mathrm{t}} \cdot \dot{\hat{\boldsymbol{F}}}_{\mathrm{e}}$,

which proves the equivalence (see also Eq. (8) 1 ). The flow rule in Eq. (19) can also be reformulated for the intermediate configuration resulting in $\hat{\boldsymbol{L}}_{\mathrm{p}}=\gamma \hat{\boldsymbol{N}}_{\hat{\phi}} \cdot \hat{\boldsymbol{C}}_{\mathrm{e}}$, where $\hat{\boldsymbol{N}}_{\hat{\phi}}=$ $\hat{\boldsymbol{F}}_{\mathrm{e}}^{-1} \cdot[\partial \hat{\phi} / \partial \hat{\boldsymbol{\tau}}] \cdot \hat{\boldsymbol{F}}_{\mathrm{e}}^{-\mathrm{t}}$ is the normal to the surface yield surface in the intermediate configuration. The spatial counterpart of the normal $\hat{\boldsymbol{N}}_{\hat{\phi}}$ is denoted by $\hat{\boldsymbol{n}}_{\hat{\phi}}=\partial \hat{\phi} / \partial \hat{\boldsymbol{\tau}}$. Furthermore, a pressure-independent surface yield condition implies an isochoric plastic deformation, i.e $\hat{J}_{\mathrm{p}}=1$, as follows: first from $\hat{J}=\hat{J}_{\mathrm{e}} \hat{J}_{\mathrm{p}}$ we have

$$
\begin{aligned}
\ln \hat{J} & =\ln \left(\hat{J}_{\mathrm{e}} \hat{J}_{\mathrm{p}}\right) \Longrightarrow \frac{\mathrm{D} \ln \hat{J}}{\mathrm{D} t} \\
& =\frac{\mathrm{D} \ln \hat{J}_{\mathrm{e}}}{\mathrm{D} t}+\frac{\mathrm{D} \ln \hat{J}_{\mathrm{p}}}{\mathrm{D} t} \Longrightarrow \frac{\dot{\hat{J}}}{\hat{J}}=\frac{\dot{\hat{J}}_{\mathrm{e}}}{\hat{J}_{\mathrm{e}}}+\frac{\dot{\hat{J}}_{\mathrm{p}}}{\hat{J}_{\mathrm{p}}}
\end{aligned}
$$

Next expanding the time derivative of $\dot{\hat{J}}_{\mathrm{e}}$ results in ${ }^{6}$

$$
\begin{aligned}
\dot{\hat{J}}_{\mathrm{e}} & =\hat{J}_{\mathrm{e}} \widehat{\operatorname{trace}}\left(\hat{\boldsymbol{d}}-\gamma \hat{\boldsymbol{n}}_{\hat{\phi}}\right) \quad \text { with } \dot{\hat{J}} \\
& =\hat{J} \widehat{\operatorname{trace}}(\hat{\boldsymbol{d}}) \Longrightarrow \frac{\mathrm{D} \ln \hat{J}_{\mathrm{p}}}{\mathrm{D} t}=\gamma \widehat{\operatorname{trace}} \hat{\boldsymbol{n}}_{\hat{\phi}} .
\end{aligned}
$$

Therefore if $\widehat{\operatorname{trace}} \hat{\boldsymbol{n}}_{\hat{\phi}}=0$, then $\hat{J}_{\mathrm{p}}=1$.

\subsection{Von Mises-type surface yield criterion and the case of decoupled surface volumetric-deviatoric response}

In this section we consider the von Mises-type surface yield condition $^{7}$ as a function of the surface Kirchhoff stress tensor as

$\hat{\phi}\left(\hat{\boldsymbol{\tau}}, \hat{F}_{\mathrm{p}}\right):=\|\widehat{\operatorname{dev}}(\hat{\boldsymbol{\tau}})\|-\sqrt{\frac{2}{3}}\left[\hat{\sigma}_{\mathrm{Y}}+\hat{K}\left(\hat{F}_{\mathrm{p}}\right)\right] \leq 0$,

where $\hat{\sigma}_{\mathrm{Y}}$ denotes the surface yield stress, $\hat{K}$ is a (non)linear function of $\hat{F}_{\mathrm{p}}$, the surface hardening variable, which deter-

\footnotetext{
6 The surface trace operator for spatial second order tensor is defined as $\widehat{\operatorname{trace}}\{\hat{\boldsymbol{\bullet}}\}=\{\hat{\boldsymbol{\bullet}}\}: \hat{\boldsymbol{i}}$. In the material configuration the surface trace operator is defined correspondingly as $\widehat{\operatorname{Trace}}\{\hat{\boldsymbol{\bullet}}\}=\{\hat{\boldsymbol{\bullet}}\}: \hat{\boldsymbol{I}}$.

${ }^{7}$ Note that henceforth only the classical example for metal plasticity, i.e. the von Mises-type yield criterion is considered. Thus, we only take into account the simplest plasticity model, i.e. $\mathrm{J}_{2}$ type flow theory with isotropic hardening to be developed on the surface. This is to motivate a surface elastoplasticity model and examine its computational aspects.
} 
mines the isotropic hardening behavior of the surface and $\widehat{\operatorname{dev}}\{\hat{\bullet}\}=\{\hat{\bullet}\}-\frac{1}{2} \widehat{\operatorname{trace}}\{\hat{\bullet}\} \hat{\boldsymbol{i}}$ is the spatial surface deviatoric operator. The factor $\sqrt{2 / 3}$ is used for the sake of analogy with classical bulk von Mises yield criterion.

Next we introduce briefly the kinematics of the surface deviatoric-volumetric multiplicative split according to the chosen yield criterion Eq. (23). Let $\hat{\boldsymbol{F}}^{\text {iso }}$ and $\hat{\boldsymbol{F}}^{\text {vol }}$ denote the volume-preserving (angle-changing) and volumetric part of the surface deformation gradient ${ }^{8}$, hence $\hat{\boldsymbol{F}}=\hat{\boldsymbol{F}}^{\text {vol }} \cdot \hat{\boldsymbol{F}}^{\text {iso }}$ where $\widehat{\operatorname{det}} \hat{\boldsymbol{F}}^{\text {iso }}=\hat{\boldsymbol{J}}^{\text {iso }}=1$. Consequently $\hat{\boldsymbol{F}}^{\text {iso }}, \hat{\boldsymbol{F}}^{\text {vol }}, \hat{\boldsymbol{C}}^{\text {iso }}$ and $\hat{\boldsymbol{b}}^{\text {iso }}$ are defined as

$\hat{\boldsymbol{F}}^{\text {iso }}:=\hat{J}^{-1 / 2} \hat{\boldsymbol{F}}, \quad \hat{\boldsymbol{F}}^{\mathrm{vol}}:=\hat{J}^{1 / 2} \hat{\boldsymbol{I}}$,

$\hat{\boldsymbol{C}}^{\text {iso }}:=\left[\hat{\boldsymbol{F}}^{\text {iso }}\right]^{\mathrm{t}} \cdot \hat{\boldsymbol{F}}^{\text {iso }} \equiv \hat{\boldsymbol{J}}^{-1} \hat{\boldsymbol{C}}$ and

$\hat{\boldsymbol{b}}^{\text {iso }}:=\hat{\boldsymbol{F}}^{\text {iso }} \cdot\left[\hat{\boldsymbol{F}}^{\text {iso }}\right]^{\mathrm{t}} \equiv \hat{\boldsymbol{J}}^{-1} \hat{\boldsymbol{b}}$.

Note that the same volumetric-isochoric decoupling can be applied on the plastic and elastic contribution of any of the above strain measures. We also point out that the exponents $-1 / 2,1 / 2$ and -1 appearing in Eq. (24) are due to the lower-dimensional nature of the surface. The corresponding exponents in the bulk assume the familiar values $-1 / 3,1 / 3$ and $-2 / 3$.

\subsection{Model problem: decoupled hyperelastic stress response}

As a model problem, and as the basis for the numerical examples ${ }^{9}$, we consider the following decoupled surface free energy

$\hat{\Psi}=\hat{\Psi}^{\text {iso }}\left(\hat{\boldsymbol{b}}_{\mathrm{e}}^{\mathrm{iso}}\right)+\hat{\Psi}^{\mathrm{vol}}\left(\hat{J}_{\mathrm{e}}\right)$ with

$\hat{\Psi}^{\text {iso }}=\frac{1}{2} \hat{\mu}\left[\widehat{\operatorname{trace}} \hat{b}_{\mathrm{e}}^{\text {iso }}-2\right]$ and

$\hat{\Psi}^{\mathrm{vol}}=\frac{1}{2} \hat{\kappa}\left[\frac{1}{2}\left[\hat{J}_{\mathrm{e}}^{2}-1\right]-\ln \hat{J}_{\mathrm{e}}\right]$,

where $\hat{\Psi}^{\text {iso }}\left(\hat{\boldsymbol{b}}_{\mathrm{e}}\right)$ and $\hat{\Psi}^{\text {vol }}\left(\hat{J}_{\mathrm{e}}\right)$ are the isochoric and volumetric contribution to the total surface free energy and $\hat{\boldsymbol{b}}_{\mathrm{e}}^{\text {iso }}=\hat{J}_{\mathrm{e}}^{-1} \hat{\boldsymbol{b}}_{\mathrm{e}}$. The surface shear modulus and surface bulk modulus are denoted respectively by $\hat{\mu}$ and $\hat{\kappa}$. Next, to obtain the surface

\footnotetext{
8 The term volumetric has a different meaning on the surface. A surface volumetric deformation describes a deformation that changes the area. A volumetric deformation in the bulk however changes the volume. Nonetheless, we use the same term for both the bulk and the surface for the sake of simplicity.

${ }^{9}$ We mention the assumptions made for the numerical part of the current manuscript: first, the surface stress response is isotropic. Second, the plastic spin on the surface is assumed to vanish. Third, the main focus here is on metal plasticity meaning that plastic yielding is isochoric, i.e. $\hat{J}_{\mathrm{p}}=1$, which justifies the decoupling of the surface strain energy. Note that the same assumptions are also made for the bulk elastoplasticity.
}

Kirchhoff stress $\hat{\boldsymbol{\tau}}$ from the energy given above we first note that

$\widehat{\operatorname{trace}} \hat{\boldsymbol{b}}_{\mathrm{e}}^{\text {iso }}=\widehat{\operatorname{Trace}} \hat{\boldsymbol{C}}_{\mathrm{e}}^{\text {iso }}$ or $\widehat{\operatorname{trace}} \hat{\boldsymbol{b}}_{\mathrm{e}}^{\text {iso }}=\hat{\boldsymbol{C}}^{\text {iso }}:\left[\hat{\boldsymbol{C}}_{\mathrm{p}}^{\text {iso }}\right]^{-1}$ with

$\hat{\boldsymbol{C}}_{\mathrm{e}}^{\mathrm{iso}}=\hat{\boldsymbol{J}}_{\mathrm{e}}^{-1} \hat{\boldsymbol{C}}_{\mathrm{e}}, \quad \hat{J}_{\mathrm{e}}=\left[\widehat{\operatorname{Det}} \hat{\boldsymbol{C}}_{\mathrm{e}}\right]^{1 / 2}$

Subsequently using Eq. (26) one can re-parameterize Eq. (25) in material quantities. Consequently the surface Kirchhoff stress reads

$$
\begin{aligned}
\hat{\boldsymbol{\tau}} & =2 \hat{\boldsymbol{F}}_{\mathrm{e}} \cdot \frac{\partial \hat{\Psi}}{\partial \hat{\boldsymbol{C}}_{\mathrm{e}}} \cdot \hat{\boldsymbol{F}}_{\mathrm{e}}^{\mathrm{t}}=2 \hat{\boldsymbol{F}} \cdot \frac{\partial \hat{\Psi}\left(\hat{\boldsymbol{C}}, \hat{\boldsymbol{C}}_{\mathrm{p}}\right)}{\partial \hat{\boldsymbol{C}}} \cdot \hat{\boldsymbol{F}}^{\mathrm{t}} \\
& =\underbrace{\frac{1}{2} \hat{\kappa}\left[\hat{\boldsymbol{J}}_{\mathrm{e}}^{2}-1\right] \hat{\boldsymbol{i}}}_{\hat{\boldsymbol{\tau}}^{\text {vol }}}+\underbrace{\hat{\mu} \widehat{\operatorname{dev}} \hat{\boldsymbol{b}}_{\mathrm{e}}^{\text {iso }}}_{\hat{\boldsymbol{\tau}}^{\text {iso }}},
\end{aligned}
$$

where the intermediate steps to derive Eq. (27) are given as

$$
\begin{aligned}
\hat{\boldsymbol{\tau}}^{\mathrm{vol}} & =\frac{1}{2} \kappa \hat{\boldsymbol{F}}_{\mathrm{e}} \cdot\left[\left[\hat{J}_{\mathrm{e}}^{2}-1\right] \hat{\boldsymbol{C}}_{\mathrm{e}}^{-1}\right] \cdot \hat{\boldsymbol{F}}_{\mathrm{e}}^{\mathrm{t}} \text { and } \\
\hat{\boldsymbol{\tau}}^{\mathrm{iso}} & =\hat{\mu} \hat{J}_{\mathrm{e}}^{-1} \hat{\boldsymbol{F}} \cdot\left[-\frac{1}{2} \hat{\boldsymbol{C}}^{-1}\left[\hat{\boldsymbol{C}}:\left(\hat{\boldsymbol{C}}_{\mathrm{p}}^{\mathrm{iso}}\right)^{-1}\right]+\left(\hat{\boldsymbol{C}}_{\mathrm{p}}^{\mathrm{iso}}\right)^{-1}: \hat{\mathbb{I}}^{\mathrm{sym}}\right] \cdot \hat{\boldsymbol{F}}^{\mathrm{t}} \\
& =\hat{\mu} \hat{J}_{\mathrm{e}}^{-1} \hat{\boldsymbol{F}} \cdot\left[\widehat{\operatorname{Dev}}\left(\hat{\boldsymbol{C}}_{\mathrm{p}}^{\mathrm{iso}}\right)^{-1}\right] \cdot \hat{\boldsymbol{F}}^{\mathrm{t}}
\end{aligned}
$$

with $\widehat{\operatorname{Dev}}\{\hat{\bullet}\}:=\{\hat{\bullet}\}-\frac{1}{2}[\hat{\boldsymbol{C}}:\{\hat{\bullet}\}] \hat{\boldsymbol{C}}^{-1}$ being the material surface deviatoric operator and $\hat{\mathbb{I}}^{\mathrm{Sym}}=\partial \hat{\boldsymbol{C}} / \partial \hat{\boldsymbol{C}}$. Note that in the derivation above it is implied that $\hat{J}=\hat{J}_{\mathrm{e}}$ since $\hat{J}=\hat{J}_{\mathrm{e}} \hat{J}_{\mathrm{p}}$ and $\hat{J}_{\mathrm{p}}=1$. Consequently $\hat{\boldsymbol{C}}_{\mathrm{p}}^{\text {iso }}=\hat{\boldsymbol{C}}_{\mathrm{p}}$. Next the isochoric and volumetric surface elasticity tensors of the model problem Eq. (25) both in the material and spatial configurations are given as

$$
\begin{aligned}
& \hat{\mathbb{C}}_{\mathrm{e}}^{\mathrm{vol}}=2 \frac{\partial \hat{\boldsymbol{S}}^{\mathrm{vol}}}{\partial \hat{\boldsymbol{C}}_{\mathrm{e}}}=\hat{\kappa}\left[\widehat{\operatorname{det}} \hat{\boldsymbol{C}}_{\mathrm{e}} \hat{\boldsymbol{C}}_{\mathrm{e}}^{-1} \otimes \hat{\boldsymbol{C}}_{\mathrm{e}}^{-1}+\frac{\partial \hat{\boldsymbol{C}}_{\mathrm{e}}^{-1}}{\partial \hat{\boldsymbol{C}}_{\mathrm{e}}}\left[\widehat{\operatorname{det}} \hat{\boldsymbol{C}}_{\mathrm{e}}-1\right]\right] \\
& \text { with }\left[\hat{\mathbb{C}}_{\mathrm{e}}^{\mathrm{vol}}\right]_{i j k l}=\left[\hat{\boldsymbol{F}}_{\mathrm{e}}\right]_{i I}\left[\hat{\boldsymbol{F}}_{\mathrm{e}}\right]_{j J}\left[\hat{\boldsymbol{F}}_{\mathrm{e}}\right]_{k K}\left[\hat{\boldsymbol{F}}_{\mathrm{e}}\right]_{l L}\left[\hat{\mathbb{C}}_{\mathrm{e}}^{\mathrm{vol}}\right]_{I J K L} \\
& \Longrightarrow \hat{\mathbb{C}}_{\mathrm{e}}^{\mathrm{vol}}=\hat{\kappa}\left[\hat{J}_{\mathrm{e}}^{2} \hat{\boldsymbol{i}} \otimes \hat{\boldsymbol{i}}+\hat{o}^{\mathrm{sym}}\left[\hat{J}_{\mathrm{e}}^{2}-1\right]\right],
\end{aligned}
$$

and

$$
\begin{aligned}
& \hat{\mathbb{C}}_{\mathrm{e}}^{\text {iso }}=\frac{\partial \hat{\boldsymbol{S}}^{\text {iso }}}{\partial \hat{\boldsymbol{C}}}=2 \hat{\mu}\left[\left(\hat{\boldsymbol{C}}_{\mathrm{p}}^{\text {iso }}\right)^{-1}-\frac{1}{2}\left[\left(\hat{\boldsymbol{C}}_{\mathrm{p}}^{\text {iso }}\right)^{-1}: \hat{\boldsymbol{C}}\right] \hat{\boldsymbol{C}}^{-1}\right] \\
& \otimes\left[-\frac{1}{2} \hat{J}_{\mathrm{e}}^{-1} \hat{\boldsymbol{C}}^{-1}\right]+2 \hat{\mu} \hat{\boldsymbol{J}}_{\mathrm{e}}^{-1} \hat{\boldsymbol{C}}^{-1} \otimes\left[-\frac{1}{2}\left(\hat{\boldsymbol{C}}_{\mathrm{p}}^{\mathrm{iso}}\right)^{-1}: \hat{\mathbb{I}}^{\mathrm{sym}}\right] \\
& -\hat{\mu} \hat{\boldsymbol{J}}_{\mathrm{e}}^{-1} \hat{\boldsymbol{C}}:\left(\hat{\boldsymbol{C}}_{\mathrm{p}}^{\mathrm{iso}}\right)^{-1}\left[-\frac{1}{2}\left[\hat{\boldsymbol{C}}^{-1} \bar{\otimes} \hat{\boldsymbol{C}}^{-1}+\hat{\boldsymbol{C}}^{-1} \underline{\otimes} \hat{\boldsymbol{C}}^{-1}\right]\right] \\
& \text { with }\left[\hat{\mathbb{C}}_{\mathrm{e}}^{\text {iso }}\right]_{i j k l}=[\hat{\boldsymbol{F}}]_{i I}[\hat{\boldsymbol{F}}]_{j J}[\hat{\boldsymbol{F}}]_{k K}[\hat{\boldsymbol{F}}]_{l L}\left[\hat{\mathbb{C}}_{\mathrm{e}}^{\text {iso }}\right]_{I J K L} \Longrightarrow \hat{\mathbb{C}}_{\mathrm{e}}^{\text {iso }} \\
& =\hat{\mu} \widehat{\operatorname{trace}} \hat{\boldsymbol{b}}_{\mathrm{e}}^{\text {iso }}\left[\mathrm{o}^{\text {osym }}-\frac{1}{2} \hat{\boldsymbol{i}} \otimes \hat{\boldsymbol{i}}\right]-\hat{\mu}\left[\widehat{\operatorname{dev}} \hat{\boldsymbol{b}}_{\mathrm{e}}^{\text {iso }} \otimes \hat{\boldsymbol{i}}+\hat{\boldsymbol{i}} \otimes \widehat{\operatorname{dev}} \hat{\boldsymbol{b}}_{\mathrm{e}}^{\mathrm{iso}}\right] \text {, }
\end{aligned}
$$


where

$\hat{\boldsymbol{S}}^{\mathrm{vol}}=\frac{1}{2} \kappa\left[\left[\hat{\boldsymbol{J}}_{\mathrm{e}}^{2}-1\right] \hat{\boldsymbol{C}}_{\mathrm{e}}^{-1}\right]$ and $\quad \hat{\boldsymbol{S}}^{\text {iso }}=\hat{\mu} \hat{\boldsymbol{J}}_{\mathrm{e}}^{-1}\left[\widehat{\operatorname{Dev}}\left(\hat{\boldsymbol{C}}_{\mathrm{p}}^{\text {iso }}\right)^{-1}\right]$.

The volumetric surface elasticity tensors in the material and spatial configuration in the above are denoted by $\hat{\mathbb{C}}_{\mathrm{e}}^{\text {vol }}$ and $\hat{\mathbb{C}}_{\mathrm{e}}^{\mathrm{vol}}$, respectively. Their isochoric counterparts are denoted by $\hat{\mathbb{C}}_{\mathrm{e}}^{\mathrm{iso}}$ and $\hat{\mathbb{C}}_{\mathrm{e}}^{\mathrm{iso}}$, respectively.

Now Considering the definition of $\hat{\boldsymbol{\tau}}^{\text {iso }}$ in Eq. (27) and $\hat{\boldsymbol{n}}_{\hat{\phi}}=\widehat{\operatorname{dev}} \hat{\boldsymbol{\tau}} /\|\widehat{\operatorname{dev}} \hat{\boldsymbol{\tau}}\|$, one can simplify the flow rule Eq. (18) as follows ${ }^{10}$

$$
\begin{aligned}
-\frac{1}{2} £_{\hat{\boldsymbol{v}}} \hat{\boldsymbol{b}}_{\mathrm{e}} & =\gamma \hat{\boldsymbol{n}}_{\hat{\phi}} \cdot \hat{\boldsymbol{b}}_{\mathrm{e}}=\gamma \hat{J}_{\mathrm{e}} \hat{\boldsymbol{n}}_{\hat{\phi}} \cdot\left[\frac{1}{2} \widehat{\operatorname{trace}} \hat{\boldsymbol{b}}_{\mathrm{e}}^{\mathrm{iso}} \hat{\boldsymbol{i}}+\widehat{\operatorname{dev}} \hat{\boldsymbol{b}}_{\mathrm{e}}^{\mathrm{iso}}\right] \\
& =\gamma \hat{J}_{\mathrm{e}}\left[\frac{1}{2} \widehat{\operatorname{trace}} \hat{\boldsymbol{b}}_{\mathrm{e}}^{\text {iso }} \hat{\boldsymbol{n}}_{\hat{\phi}}+\hat{\boldsymbol{n}}_{\hat{\phi}} \cdot \frac{\hat{\mu} \widehat{\operatorname{dev}} \hat{\boldsymbol{b}}_{\mathrm{e}}^{\text {iso }}}{\hat{\mu}\left\|\widehat{\operatorname{dev}} \hat{\boldsymbol{b}}_{\mathrm{e}}^{\text {iso }}\right\|} \frac{\hat{\mu}\left\|\widehat{\operatorname{dev}} \hat{\boldsymbol{b}}_{\mathrm{e}}^{\text {iso }}\right\|}{\hat{\mu}}\right] \\
& =\gamma \hat{J}_{\mathrm{e}}\left[\frac{1}{2} \widehat{\operatorname{trace}} \hat{\boldsymbol{b}}_{\mathrm{e}}^{\text {iso }} \hat{\boldsymbol{n}}_{\hat{\phi}}+\hat{\boldsymbol{n}}_{\hat{\phi}}^{2} \frac{\| \widehat{\operatorname{dev}} \hat{\boldsymbol{x}}\lceil}{\hat{\mu}}\right] \\
& \approx \frac{1}{2} \gamma \hat{J}_{\mathrm{e}} \widehat{\operatorname{trace}} \hat{\boldsymbol{b}}_{\mathrm{e}}^{\text {iso }} \hat{\boldsymbol{n}}_{\hat{\phi}},
\end{aligned}
$$

with $\|\widehat{\operatorname{dev}} \hat{\boldsymbol{\tau}}\| / \hat{\mu} \cong 10^{-3}$ for metals and thus neglected. Using Eq. $(8)_{2}$ the simplified surface flow rule (the last term in Eq. (32)) can also be given in the material configuration as

$\dot{\hat{\boldsymbol{C}}}_{\mathrm{p}}^{-1}=-\gamma \widehat{\operatorname{trace}} \hat{\boldsymbol{b}}_{\mathrm{e}} \hat{\boldsymbol{F}}^{-1} \cdot \hat{\boldsymbol{n}}_{\hat{\phi}} \cdot \hat{\boldsymbol{F}}^{-\mathrm{t}}$.

Finally to complete the surface plasticity formulation, the evolution of the hardening variable $\hat{F}_{\mathrm{p}}$ in terms of the plastic multiplier (Lagrange multiplier or consistency parameter) $\gamma$, and the consistency condition are now given as follows

$\dot{\hat{F}}_{\mathrm{p}}=\sqrt{\frac{2}{3}} \gamma$ and $\gamma \dot{\hat{\phi}}\left(\hat{\boldsymbol{\tau}}, \hat{F}_{\mathrm{p}}\right)=0$.

\subsubsection{Return mapping algorithm}

In this section the time discretization of the model introduced in the previous section, i.e. the integration algorithm for $\mathbf{J}_{2}$ type plasticity on the surface together with the return mapping algorithm are given. Due to the path-dependence of the surface plasticity model, the surface stress tensor is the solution of a constitutive initial value problem meaning that the surface stress tensor is not only a function of the instantaneous value of the surface strain but also depends on the history of surface strain. Therefore an appropriate numerical algorithm for integration of the rate constitutive equations is a requirement in the finite element simulation of such models. In doing so, we assume the data $\left\{\hat{\boldsymbol{\varphi}}_{\tau},\left[\hat{\boldsymbol{b}}_{\mathrm{e}}\right]_{\tau},\left[F_{\mathrm{p}}\right]_{\tau}, \hat{\boldsymbol{F}}_{\tau}\right\}$ is

\footnotetext{
10 Alternatively, a formulation adapting the flow rule in [62] to surface plasticity is possible.
}

known at time $t_{\tau}$. Consequently the surface Kirchhoff stress tensor $\hat{\boldsymbol{\tau}}_{\tau}$ is also known through Eq. (27). We start by providing the discretized evolution Eqs. (33) and (34) 1 in the material configuration as

$$
\begin{aligned}
{\left[\hat{\boldsymbol{C}}_{\mathrm{p}}^{-1}\right]_{\tau+1}-\left[\hat{\boldsymbol{C}}_{\mathrm{p}}^{-1}\right]_{\tau}=} & -\Delta \gamma\left[\left[\hat{\boldsymbol{C}}_{\mathrm{p}}^{-1}\right]_{\tau+1}:\left[\hat{\boldsymbol{C}}^{-1}\right]_{\tau+1}\right] \hat{\boldsymbol{F}}_{\tau+1}^{-1} \\
& \cdot\left[\hat{\boldsymbol{n}}_{\hat{\phi}}\right]_{\tau+1} \cdot \hat{\boldsymbol{F}}_{\tau+1}^{-\mathrm{t}} \text { and } \\
{\left[F_{\mathrm{p}}\right]_{\tau+1}-\left[F_{\mathrm{p}}\right]_{\tau}=} & \sqrt{\frac{2}{3}} \Delta \gamma
\end{aligned}
$$

where $\tau$ denotes the time step and time discretization scheme is backward-Euler. The spatial counterpart of the above reads now

$$
\begin{aligned}
& {\left[\hat{\boldsymbol{b}}_{\mathrm{e}}^{\text {iso }}\right]_{\tau+1}=} \hat{\mathcal{F}}_{\tau+1}^{\text {iso }} \cdot\left[\hat{\boldsymbol{b}}_{\mathrm{e}}^{\text {iso }}\right]_{\tau} \cdot\left(\hat{\mathcal{F}}_{\tau+1}^{\text {iso }}\right)^{\mathrm{t}} \\
&-\Delta \gamma \widehat{\operatorname{trace}} \hat{\boldsymbol{b}}_{\tau+1}^{\text {iso }}\left[\hat{\boldsymbol{n}}_{\hat{\phi}}\right]_{\tau+1} \text { with } \\
& \hat{\mathcal{F}}_{\tau+1}^{\text {iso }}=\hat{\boldsymbol{F}}_{\tau+1}^{\text {iso }} \cdot\left(\hat{\boldsymbol{F}}_{\tau}^{\text {iso }}\right)^{-1} .
\end{aligned}
$$

Next we define a trial elastic state, based on the known data as follows:

$\left[\hat{\boldsymbol{C}}_{\mathrm{p}}^{-1}\right]_{\tau+1}^{\text {trial }}=\left[\hat{\boldsymbol{C}}_{\mathrm{p}}^{-1}\right]_{\tau}, \quad\left[\hat{F}_{\mathrm{p}}\right]_{\tau+1}^{\text {trial }}=\left[\hat{F}_{\mathrm{p}}\right]_{\tau}$,

$\left[\hat{\boldsymbol{b}}_{\mathrm{e}}^{\text {iso }}\right]_{\tau+1}^{\text {trial }}=\hat{\mathcal{F}}_{\tau+1}^{\text {iso }} \cdot\left[\hat{\boldsymbol{b}}_{\mathrm{e}}^{\text {iso }}\right]_{\tau} \cdot\left[\hat{\mathcal{F}}_{\tau+1}^{\text {iso }}\right]^{\mathrm{t}}$ and

$\hat{\boldsymbol{\tau}}_{\tau+1}^{\text {trial }}=\frac{1}{2} \hat{\kappa}\left[\left[\hat{J}_{\mathrm{e}}^{2}\right]_{\tau+1}-1\right] \hat{\boldsymbol{i}}+\hat{\mu} \widehat{\operatorname{dev}}\left[\hat{\boldsymbol{b}}_{\mathrm{e}}^{\text {iso }}\right]_{\tau+1}^{\text {trial }}$.

Having obtained the trial state, one can define a temporallydiscretized trial surface yield condition $\hat{\phi}^{\text {trial }}$, using Eq. (23) as

$$
\begin{aligned}
\hat{\phi}_{\tau+1}^{\text {trial }}= & \left.\hat{\phi}\left(\hat{\boldsymbol{\tau}}_{\tau+1}^{\text {trial }}, \hat{[} F_{\mathrm{p}}\right]_{\tau}\right):=\left\|\widehat{\operatorname{dev}} \hat{\boldsymbol{\tau}}_{\tau+1}^{\text {trial }}\right\| \\
& -\sqrt{\frac{2}{3}}\left[\hat{\sigma}_{\mathrm{Y}}+\hat{K}\left(\left[\hat{F}_{\mathrm{p}}\right]_{\tau}\right)\right]
\end{aligned}
$$

from which the following two alternatives arise:

if $\hat{\phi}_{\tau+1}^{\text {trial }} \leq 0$ trial step is elastic $\Rightarrow \Delta \gamma$

$$
=0 \quad \text { and } \quad\{\hat{\bullet}\}_{\tau+1}=\{\hat{\bullet}\}_{\tau+1}^{\text {trial }}
$$

if $\hat{\phi}_{\tau+1}^{\text {trial }}>0$ trial step is plastic $\Rightarrow \Delta \gamma$

$$
>0 \text { and }\{\hat{\boldsymbol{\bullet}}\}_{\tau+1} \neq\{\hat{\boldsymbol{\bullet}}\}_{\tau+1}^{\text {trial }} \text { and return mapping. }
$$

In case the second situation above arises, since $\Delta \gamma>0$, to find $\Delta \gamma$, we require $\hat{\phi}\left(\hat{\boldsymbol{\tau}}_{\tau+1},\left[\hat{F}_{\mathrm{p}}\right]_{\tau+1}\right)=0$. Thus we have

$$
\begin{aligned}
\hat{\phi}\left(\hat{\boldsymbol{\tau}}_{\tau+1},\left[\hat{F}_{\mathrm{p}}\right]_{\tau+1}\right)= & \left\|\widehat{\operatorname{dev}} \hat{\boldsymbol{\tau}}_{\tau+1}\right\|-\sqrt{\frac{2}{3}}\left[\hat{\sigma}_{\mathrm{Y}}+\hat{K}\left(\left[\hat{F}_{\mathrm{p}}\right]_{\tau+1}\right)\right] \\
= & \left\|\widehat{\operatorname{dev}} \hat{\boldsymbol{\tau}}_{\tau+1}^{\text {trial }}\right\|-\hat{\mu} \Delta \gamma \widehat{\operatorname{trace}}\left(\hat{\boldsymbol{b}}_{\mathrm{e}}^{\text {iso }}\right)_{\tau+1}^{\text {trial }} \\
& -\sqrt{\frac{2}{3}}\left[\hat{\sigma}_{\mathrm{Y}}+\hat{K}\left(\left[\hat{F}_{\mathrm{p}}\right]_{\tau+1}\right)\right]=0
\end{aligned}
$$


In general $\hat{\phi}\left(\hat{\boldsymbol{\tau}}_{\tau+1},\left[\hat{F}_{\mathrm{p}}\right]_{\tau+1}\right)$ is a non-linear ${ }^{11}$ function of $\Delta \gamma$, and thus to solve Eq. (40), one requires the use of NewtonRaphson method.

Remark 4 In deriving the last term in Eq. (40) we made use of the following relation

$\left\|\widehat{\operatorname{dev}} \hat{\boldsymbol{\tau}}_{\tau+1}\right\|+\hat{\mu} \Delta \gamma \widehat{\operatorname{trace}}\left(\hat{\boldsymbol{b}}_{\mathrm{e}}^{\text {iso }}\right)_{\tau+1}^{\text {trial }}=\left\|\widehat{\operatorname{dev}} \hat{\boldsymbol{\tau}}_{\tau+1}^{\text {trial }}\right\|$.

To prove the above we recall the definition of $\hat{\boldsymbol{\tau}}^{\text {iso }}$ in Eq. (27), the flow rule Eq. $(36)_{1}$, Eq. $(37)_{4}, \widehat{\operatorname{trace}} \hat{\boldsymbol{b}}_{\mathrm{e}}^{\text {iso }}=\widehat{\operatorname{trace}}\left(\hat{\boldsymbol{b}}_{\mathrm{e}}^{\text {iso }}\right)^{\text {trial }}$ and $\hat{\boldsymbol{n}}_{\hat{\phi}}=\widehat{\operatorname{dev}} \hat{\boldsymbol{\tau}} /\|\widehat{\operatorname{dev}} \hat{\boldsymbol{\tau}}\|$. We then write

$$
\begin{aligned}
\widehat{\operatorname{dev}} \hat{\boldsymbol{\tau}}_{\tau+1}= & \hat{\mu} \widehat{\operatorname{dev}}\left(\hat{\boldsymbol{b}}_{\mathrm{e}}^{\mathrm{iso}}\right)_{\tau+1}=\hat{\mu} \widehat{\operatorname{dev}}\left(\hat{\boldsymbol{b}}_{\mathrm{e}}^{\text {iso }}\right)_{\tau+1}^{\text {trial }} \\
& -\hat{\mu} \Delta \gamma \widehat{\operatorname{trace}}\left(\hat{\boldsymbol{b}}_{\mathrm{e}}^{\text {iso }}\right)_{\tau+1}^{\text {trial }}\left[\hat{\boldsymbol{n}}_{\hat{\phi}}\right]_{\tau+1} \\
& \Longrightarrow\left[\left\|\widehat{\operatorname{dev}} \hat{\boldsymbol{\tau}}_{\tau+1}\right\|+\hat{\mu} \Delta \gamma \widehat{\operatorname{trace}}\left(\hat{\boldsymbol{b}}_{\mathrm{e}}^{\text {iso }}\right)_{\tau+1}^{\text {trial }}\right]\left[\hat{\boldsymbol{n}}_{\hat{\phi}}\right]_{\tau+1} \\
= & \left\|\widehat{\operatorname{dev}} \hat{\boldsymbol{\tau}}_{\tau+1}^{\text {trial }}\right\|\left[\hat{\boldsymbol{n}}_{\hat{\phi}}\right]_{\tau+1}^{\text {trial }} .
\end{aligned}
$$

From the last term above it is implied that

$\left\|\widehat{\operatorname{dev}} \hat{\boldsymbol{\tau}}_{\tau+1}\right\|+\hat{\mu} \Delta \gamma \widehat{\operatorname{trace}}\left(\hat{\boldsymbol{b}}_{\mathrm{e}}^{\text {iso }}\right)_{\tau+1}^{\text {trial }}=\left\|\widehat{\operatorname{dev}} \hat{\boldsymbol{\tau}}_{\tau+1}^{\text {trial }}\right\|$ and

$\left[\hat{\boldsymbol{n}}_{\hat{\phi}}\right]_{\tau+1}=\left[\hat{\boldsymbol{n}}_{\hat{\phi}}\right]_{\tau+1}^{\text {trial }}$,

where Eq. (43) 1 concludes the proof.

Having found $\Delta \gamma$, we finally provide the update formula for $\left[\hat{\boldsymbol{b}}_{\mathrm{e}}^{\text {iso }}\right]_{\tau+1}$ and $\hat{\boldsymbol{\tau}}_{\tau+1}$ as follows

$\left[\hat{\boldsymbol{b}}_{\mathrm{e}}^{\text {iso }}\right]_{\tau+1}=\left[\hat{\boldsymbol{b}}_{\mathrm{e}}^{\text {iso }}\right]_{\tau+1}^{\text {trial }}-\Delta \gamma \widehat{\operatorname{trace}}\left(\hat{\boldsymbol{b}}_{\mathrm{e}}^{\text {iso }}\right)_{\tau+1}^{\text {trial }}\left[\hat{\boldsymbol{n}}_{\hat{\phi}}\right]_{\tau+1}$ and

$\hat{\boldsymbol{\tau}}_{\tau+1}=\hat{\boldsymbol{\tau}}_{\tau+1}^{\mathrm{vol}}+\hat{\mu} \widehat{\operatorname{dev}}\left(\hat{\boldsymbol{b}}_{\mathrm{e}}^{\text {iso }}\right)_{\tau+1}^{\text {trial }}-\Delta \gamma \hat{\mu} \widehat{\operatorname{trace}}\left(\hat{\boldsymbol{b}}_{\mathrm{e}}^{\text {iso }}\right)_{\tau+1}^{\text {trial }}\left[\hat{\boldsymbol{n}}_{\hat{\phi}}\right]_{\tau+1}$.

\subsubsection{Algorithmic elastoplastic tangent modulus}

The objective of this section is to exactly linearize the update formula provided for the surface Kirchhoff stress in Eq. (44) in order to obtain quadratic convergence associated with the Newton-Raphson method. The linearization of the first two terms in Eq. (44) 2 are given by Eqs. 29 and (30), respectively. To linearize the last term in Eq. $(44)_{2}$ we make use of Eq. $(40)_{3}$ and the following ${ }^{12}$

$$
\begin{aligned}
& \widehat{\operatorname{dev}} \hat{\boldsymbol{\tau}}=\hat{\boldsymbol{F}} \cdot \widehat{\operatorname{Dev}} \hat{\boldsymbol{S}} \cdot \hat{\boldsymbol{F}}^{\mathrm{t}}, \quad \hat{\boldsymbol{\tau}}=\hat{\boldsymbol{F}} \cdot \hat{\boldsymbol{S}} \cdot \hat{\boldsymbol{F}}^{\mathrm{t}}, \\
& \widehat{\operatorname{trace}}\left(\hat{\boldsymbol{b}}_{\mathrm{e}}^{\text {iso }}\right)_{\tau+1}^{\text {trial }}=\hat{J}^{-1} \hat{\boldsymbol{C}}:\left(\hat{\boldsymbol{C}}^{\text {iso }}\right)_{\mathrm{p}}^{-1} \text { and }
\end{aligned}
$$

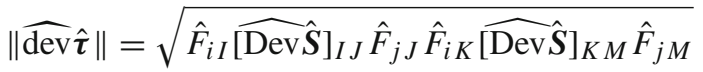

$$
\begin{aligned}
& =\sqrt{\widehat{[\operatorname{Dev} \hat{S}}]_{I J}[\widehat{\operatorname{Dev}} \hat{S}]_{K M} \hat{C}_{I K} \hat{C}_{J M}} .
\end{aligned}
$$

11 This is the case when $\hat{K}\left(\left[\hat{F}_{\mathrm{p}}\right]_{\tau+1}\right)$ is a non-linear function of $\left[\hat{F}_{\mathrm{p}}\right]_{\tau+1}$ and consequently a non-linear function of $\Delta \gamma$ since $\left[\hat{F}_{\mathrm{p}}\right]_{\tau+1}=\left[\hat{F}_{\mathrm{p}}\right]_{\tau}+$ $\sqrt{2 / 3} \Delta \gamma$.

12 In the following derivations we drop the super- and sub-index trial and $\tau+1$ for the sake of brevity.
Using the chain rule and Eq. (46) 2 , the linearization of $\|\widehat{\operatorname{dev}} \hat{\boldsymbol{\tau}}\|$ now reads

$$
\begin{aligned}
2 \frac{\partial\|\widehat{\operatorname{dev}} \hat{\boldsymbol{\tau}}\|}{\partial \hat{\boldsymbol{C}}_{A B}}= & {\left[\hat{\mathbb{C}}_{\mathrm{e}}^{\mathrm{iso}}\right]_{I J A B}\left[\hat{N}_{\hat{\phi}}\right]_{K M} \hat{\boldsymbol{C}}_{I K} \hat{\boldsymbol{C}}_{J M} } \\
& +2\|\widehat{\operatorname{Dev}} \hat{\boldsymbol{S}}\|\left[\hat{N}_{\hat{\phi}}\right]_{A I}\left[\hat{N}_{\hat{\phi}}\right]_{B J} \hat{\boldsymbol{C}}_{I J} \| \widehat{\operatorname{dev} \hat{\boldsymbol{\tau}} \|_{\mathrm{Lin}}} \\
= & 2 \hat{\boldsymbol{F}} \cdot \frac{\partial\|\widehat{\operatorname{dev}} \hat{\boldsymbol{\tau}}\|}{\partial \hat{\boldsymbol{C}}} \cdot \hat{\boldsymbol{F}}^{\mathrm{t}} \\
= & \hat{\mu} \widehat{\operatorname{trace}} \hat{\boldsymbol{b}}_{\mathrm{e}}^{\mathrm{iso}} \hat{\boldsymbol{n}}_{\hat{\phi}}+2 \widehat{\operatorname{dev}}\left(\hat{\boldsymbol{n}}_{\hat{\phi}} \cdot \widehat{\operatorname{dev}} \hat{\boldsymbol{b}}_{\mathrm{e}}^{\mathrm{iso}}\right) \\
= & \hat{\mu} \widehat{\operatorname{trace}} \hat{\boldsymbol{b}}_{\mathrm{e}}^{\mathrm{iso}} \hat{\boldsymbol{n}}_{\hat{\phi}}+2\|\widehat{\operatorname{dev}} \hat{\boldsymbol{\tau}}\| \widehat{\operatorname{dev}}\left(\hat{\boldsymbol{n}}_{\hat{\phi}} \cdot \hat{\boldsymbol{n}}_{\hat{\phi}}\right)
\end{aligned}
$$

Thus, the linearization of $\hat{\boldsymbol{n}}_{\hat{\phi}}$ becomes

$$
\begin{aligned}
{\left[\hat{\boldsymbol{n}}_{\hat{\phi}}\right]_{\mathrm{Lin}}=} & \widehat{\mathbb{C}}_{\mathrm{e}}^{\text {iso }} /\|\widehat{\operatorname{dev}} \hat{\boldsymbol{\tau}}\|-\frac{1}{\|\widehat{\operatorname{dev}} \hat{\boldsymbol{\tau}}\|} \hat{\boldsymbol{n}}_{\hat{\phi}} \\
& \otimes\left[\hat{\mu} \widehat{\operatorname{trace}} \hat{\boldsymbol{b}}_{\mathrm{e}}^{\text {iso }} \hat{\boldsymbol{n}}_{\hat{\phi}}+2\|\widehat{\operatorname{dev}} \hat{\boldsymbol{\tau}}\| \widehat{\operatorname{dev}}\left(\hat{\boldsymbol{n}}_{\hat{\phi}} \cdot \hat{\boldsymbol{n}}_{\hat{\phi}}\right)\right] .
\end{aligned}
$$

Next the linearization of Eq. (46) 3 reads

$\left[\widehat{\operatorname{trace}} \hat{\boldsymbol{b}}_{\mathrm{e}}^{\mathrm{iso}}\right]_{\mathrm{Lin}}=2 \hat{\boldsymbol{F}} \cdot \frac{\partial\left(\hat{J}^{-1} \hat{\boldsymbol{C}}_{\mathrm{p}}^{-1}: \hat{\boldsymbol{C}}\right)}{\partial \hat{\boldsymbol{C}}} \cdot \hat{\boldsymbol{F}}^{\mathrm{t}}=2 \widehat{\operatorname{dev}} \hat{\boldsymbol{b}}_{\mathrm{e}}^{\mathrm{iso}}$

What remains now is to linearize $\Delta \gamma$. By using Eq. (40) 3 and the results above we have

$$
\begin{aligned}
& \|\widehat{\operatorname{dev}} \hat{\boldsymbol{\tau}}\|_{\text {Lin }}-\hat{\mu}\left[[\Delta \gamma]_{\text {Lin }} \widehat{\operatorname{trace}} \hat{\boldsymbol{b}}_{\mathrm{e}}^{\text {iso }}+\Delta \gamma\left[\widehat{\operatorname{trace}} \hat{\boldsymbol{b}}_{\mathrm{e}}^{\text {iso }}\right]_{\text {Lin }}\right]-\frac{2}{3}[\Delta \gamma]_{\mathrm{Lin}} \frac{\mathrm{d} \hat{K}\left(\hat{F}_{\mathrm{p}}\right)}{\mathrm{d} \hat{F}_{\mathrm{p}}} \\
& =0 \Longrightarrow[\Delta \gamma]_{\text {Lin }}=\frac{\|\widehat{\operatorname{dev}} \hat{\boldsymbol{\tau}}\|_{\text {Lin }}-\hat{\mu} \Delta \gamma\left[\widehat{\operatorname{trace}} \hat{\boldsymbol{b}}_{\mathrm{e}}^{\text {iso }}\right]_{\text {Lin }}}{\hat{\mu} \widehat{\operatorname{trace}} \hat{\boldsymbol{b}}_{\mathrm{e}}^{\text {iso }}+\frac{2}{3} \frac{\mathrm{d} \hat{K}\left(\hat{F}_{\mathrm{p}}\right)}{\mathrm{d} \hat{F}_{\mathrm{p}}}} \Longrightarrow[\Delta \gamma]_{\text {Lin }} \\
& =\frac{\left[\hat{\mu} \widehat{\operatorname{trace}} \hat{\boldsymbol{b}}_{\mathrm{e}}^{\text {iso }} \hat{\boldsymbol{n}}_{\hat{\phi}}+2\|\widehat{\operatorname{dev}} \hat{\boldsymbol{\tau}}\| \widehat{\operatorname{dev}}\left(\hat{\boldsymbol{n}}_{\hat{\phi}} \cdot \hat{\boldsymbol{n}}_{\hat{\phi}}\right)\right]-2 \hat{\mu} \Delta \gamma \widehat{\operatorname{dev}} \hat{\boldsymbol{b}}_{\mathrm{e}}^{\text {iso }}}{\hat{\mu} \widehat{\operatorname{trace}} \hat{\boldsymbol{b}}_{\mathrm{e}}^{\text {iso }}+\frac{2}{3} \frac{\mathrm{d} \hat{K}\left(\hat{F}_{\mathrm{p}}\right)}{\mathrm{d} \hat{F}_{\mathrm{p}}}} .
\end{aligned}
$$

Note that in the derivations above $\|\widehat{\operatorname{dev}} \hat{\tau}\|_{\operatorname{Lin}},[\Delta \gamma]_{\operatorname{Lin}}$ and $\left[\widehat{\operatorname{trace}} \hat{\boldsymbol{b}}_{\mathrm{e}}^{\text {iso }}\right]_{\text {Lin }}$ are vectors and $\left[\hat{\boldsymbol{n}}_{\hat{\phi}}\right]_{\text {Lin }}$ is a fourth-order tensor. Having found the linearization of the terms appearing in the update formula of the stress Eq. (44) 2 , the surface algorithmic elastoplastic tangent modulus $\hat{\mathbb{C}}_{\mathrm{ep}}^{\mathrm{alg}}$ is now given as

$$
\begin{aligned}
& \hat{\mathbb{C}}_{\mathrm{ep}}^{\mathrm{alg}}=\hat{\mathbb{C}}_{\mathrm{e}}^{\mathrm{vol}}+\hat{\mathbb{C}}_{\mathrm{e}}^{\mathrm{iso}}-\Delta \gamma \hat{\mu} \widehat{\text { trace }} \hat{\boldsymbol{b}}_{\mathrm{e}}^{\mathrm{iso}}
\end{aligned}
$$

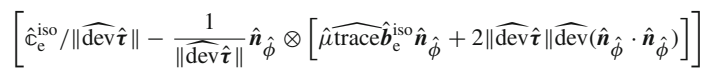

$$
\begin{aligned}
& -2 \Delta \gamma \hat{\mu} \hat{\boldsymbol{n}}_{\hat{\phi}} \otimes \widehat{\operatorname{dev}} \hat{b}_{\mathrm{e}}^{\text {iso }}-\hat{\mu} \widehat{\operatorname{trace}} \hat{\boldsymbol{b}}_{\mathrm{e}}^{\text {iso }} \\
& \frac{\left[\hat{\mu} \widehat{\operatorname{trace}} \hat{\boldsymbol{b}}_{\mathrm{e}}^{\mathrm{is}} \hat{\boldsymbol{n}}_{\hat{\phi}} \otimes \hat{\boldsymbol{n}}_{\hat{\phi}}+2\|\widehat{\operatorname{dev}} \hat{\boldsymbol{\tau}}\| \operatorname{sym}\left(\hat{\boldsymbol{n}}_{\hat{\phi}} \otimes \widehat{\operatorname{dev}}\left(\hat{\boldsymbol{n}}_{\hat{\phi}} \cdot \hat{\boldsymbol{n}}_{\hat{\phi}}\right)\right)\right]-2 \hat{\mu} \Delta \gamma \hat{\boldsymbol{n}}_{\hat{\phi}} \otimes \widehat{\operatorname{dev}} \hat{\boldsymbol{b}}_{\mathrm{e}}^{\text {iso }}}{\widehat{\mu} \widehat{\mu} \widehat{\operatorname{trace}} \hat{\boldsymbol{b}}_{\mathrm{e}}^{\text {iso }}+\frac{2}{3} \frac{\mathrm{d} \hat{K}\left(\hat{F}_{\mathrm{p}}\right)}{\mathrm{d} \hat{F}_{\mathrm{p}}}},
\end{aligned}
$$

where $\operatorname{sym}(\bullet)$ is the major symmetrization operator. 


\section{Computational framework}

In this section we establish a numerical framework that encompasses elastoplasticity of surfaces. For further details of the finite element implementation on surfaces see [18$22]$. The weak form, together with its temporal and spatial discretizations will be presented next. The localized force balance equations in the bulk and on the surface given in Table 1 are tested with vector valued test functions $\delta \varphi \in$ $\mathscr{H}^{1}\left(\mathcal{B}_{0}\right)$ and $\delta \hat{\boldsymbol{\varphi}} \in \mathscr{H}^{1}\left(\mathcal{S}_{0}\right)$, respectively. By integrating the result over all domains in the material configuration, using the bulk and surface divergence theorems and the superficiality properties of the surface Piola stress, the weak form of the balance of linear momentum reads

$$
\begin{aligned}
& \int_{\mathcal{B}_{0}} \boldsymbol{P}: \operatorname{Grad} \delta \boldsymbol{\varphi} \mathrm{d} V+\int_{\mathcal{S}_{0}} \hat{\boldsymbol{P}}: \widehat{\operatorname{Grad}} \delta \hat{\boldsymbol{\varphi}} \mathrm{d} A \\
& -\int_{\mathcal{B}_{0}} \delta \boldsymbol{\varphi} \cdot \boldsymbol{B}^{\mathrm{p}} \mathrm{d} V-\int_{\mathcal{S}_{0}} \delta \hat{\boldsymbol{\varphi}} \cdot \hat{\boldsymbol{B}}^{\mathrm{p}} \mathrm{d} A=0, \\
& \forall \delta \boldsymbol{\varphi} \in \mathscr{H}^{1}\left(\mathcal{B}_{0}\right), \quad \forall \delta \hat{\boldsymbol{\varphi}} \in \mathscr{H}^{1}\left(\mathcal{S}_{0}\right) .
\end{aligned}
$$

Since the surface stress derived in the previous section is the surface Kirchhoff stress, in the weak form formulation, relation Eq. (12) $)_{3}$ is used to convert $\hat{\boldsymbol{\tau}}$ to $\hat{\boldsymbol{P}}$.

In what follows, a classical Euler-backward integration scheme is employed. Next, the spatial discretization of the problem domain is performed using the Bubnov-Galerkin finite element method. In order to have a straightforward and efficient implementation of the finite element method, the surface elements are chosen to be consistent with the bulk elements. For example, if the bulk is discretized using triquadratic elements, then biquadratic surface elements are used. This choice has the advantage that facets to which only one bulk element is attached can be regarded as a surface element.

The domains $\mathcal{B}_{0}$ and $\mathcal{S}_{0}$ are discretized into a set of bulk and surface elements

$\mathcal{B}_{0}^{\mathrm{h}} \approx \bigcup_{\beta=1}^{n_{\mathrm{Bel}}} \mathcal{B}_{0}^{\beta}$ and $\mathcal{S}_{0}^{\mathrm{h}} \approx \bigcup_{\gamma=1}^{n_{\mathrm{Sel}}} \mathcal{S}_{0}^{\gamma}$,

where $n_{\mathrm{Bel}}$ and $n_{\text {Sel }}$ denote the number of bulk and surface elements, respectively. The geometry of the bulk and surface are approximated as a function of the natural coordinates $\xi \in[-1,1]^{3}$ and $\hat{\xi} \in[-1,1]^{2}$ assigned to the bulk and the surface, respectively, using standard interpolations according to the isoparametric concept as follows:

$$
\left.\boldsymbol{X}\right|_{\mathcal{B}_{0}^{\beta}} \approx \boldsymbol{X}^{\mathrm{h}}(\xi)=\sum_{i=1}^{n_{\mathrm{nB}}} N^{i}(\xi) \boldsymbol{X}^{i},
$$

$$
\begin{gathered}
\left.\hat{\boldsymbol{X}}\right|_{\mathcal{S}_{0}^{\gamma}} \approx \hat{\boldsymbol{X}}^{\mathrm{h}}(\hat{\xi})=\sum_{i=1}^{n_{\mathrm{nS}}} \hat{N}^{i}(\hat{\xi}) \hat{\boldsymbol{X}}^{i}, \\
\left.\boldsymbol{\varphi}\right|_{\mathcal{B}_{0}^{\beta}} \approx \boldsymbol{\varphi}^{\mathrm{h}}(\xi)=\sum_{i=1}^{n_{\mathrm{nB}}} N^{i}(\boldsymbol{\xi}) \boldsymbol{\varphi}^{i}, \\
\left.\hat{\boldsymbol{\varphi}}\right|_{\mathcal{S}_{0}^{\gamma}} \approx \hat{\boldsymbol{\varphi}}^{\mathrm{h}}(\hat{\xi})=\sum_{i=1}^{n_{\mathrm{nS}}} \hat{N}^{i}(\hat{\xi}) \hat{\boldsymbol{\varphi}}^{i},
\end{gathered}
$$

where the shape functions of the bulk and surface elements at a local node $i$ are denoted as $N^{i}$ and $\hat{N}^{i}$, respectively. The bulk and surface elements consist of $n_{\mathrm{nB}}$ and $n_{\mathrm{nS}}$ nodes respectively. The numerical integration in the bulk and on the surface is performed using Gaussian quadrature formula, see [19] for further details.

Remark 5 The surface is a two-dimensional manifold in the three-dimensional space and therefore can be described by two surface coordinates. The corresponding tangent vectors to the coordinate lines i.e. the covariant surface basis vectors are obtained by taking the derivative of the position vector on the surface with respect to the coordinates. The covariant basis vectors furnish the normal to the surface via a vector product. The surface normal is then normalized by its magnitude to obtain the unit normal to the surface, see $[36,64]$.

Now the fully discrete (spatially and temporally) form of mechanical residual associated with the global node $I$ is defined by

$$
\begin{aligned}
{\left[{ }^{\mathrm{tot}} \mathbf{R}^{I}\right]_{\tau+1}=} & \int_{\mathcal{B}_{0}} \boldsymbol{P}_{\tau+1} \cdot \operatorname{Grad} N^{I} \mathrm{~d} V-\int_{\mathcal{B}_{0}} N^{I} \boldsymbol{B}_{\tau+1}^{\mathrm{p}} \mathrm{d} V \\
& +\int_{\mathcal{S}_{0}} \hat{\boldsymbol{P}}_{\tau+1} \cdot \widehat{\operatorname{Grad}} \hat{N}^{I} \mathrm{~d} A-\int_{\mathcal{S}_{0}} \hat{N}^{I} \hat{\boldsymbol{B}}_{\tau+1}^{\mathrm{p}} \mathrm{d} A .
\end{aligned}
$$

To solve Eq. (55), a Newton-Raphson scheme is utilized, which results in the introduction of algorithmic stiffness matrix in the bulk and on the surface, respectively, as follows:

$$
\begin{aligned}
& \mathbf{K}^{I J}=\frac{\partial \mathbf{R}^{I}}{\partial \varphi^{J}}=\int_{\mathcal{B}_{0}} \operatorname{Grad} N^{I} \cdot \mathbb{A}_{\tau+1} \cdot \operatorname{Grad} N^{J} \mathrm{~d} V \text { and, } \\
& \hat{\mathbf{K}}^{I J}=\frac{\partial \hat{\mathbf{R}}^{I}}{\partial \hat{\boldsymbol{\varphi}}^{J}}=\int_{\mathcal{S}_{0}} \widehat{\operatorname{Grad}} \hat{N}^{I}: \hat{\mathbb{A}}_{\tau+1} \cdot \widehat{\operatorname{Grad}} \hat{N}^{J} \mathrm{~d} A,
\end{aligned}
$$

where $\mathbb{A}=\partial_{\boldsymbol{F}} \boldsymbol{P}$ and $\hat{\mathbb{A}}=\partial_{\hat{\boldsymbol{F}}} \hat{\boldsymbol{P}}$. The spatial surface elastoplastic tangent modulus $\hat{\mathbb{C}}_{\mathrm{ep}}^{\text {alg }}$ derived in the previous section can be connected to $\hat{\mathbb{A}}$, in index notation, using the relation

$\hat{A}_{a B c D}=\hat{F}_{B b}^{-1}\left[\left[\hat{c}_{\mathrm{cp}}^{\mathrm{alg}}\right]_{a b c d}+\hat{\tau}_{a c} \delta_{b d}\right] \hat{F}_{D d}^{-1}$. 
Table 2 Material properties assumed in the numerical examples

\begin{tabular}{lllll}
\hline & Bulk & & \multicolumn{3}{l}{ Surface } \\
\hline Lamé constant & $\mu$ & $80193.8 \mathrm{~N} / \mathrm{mm}^{2}$ & $\hat{\mu}$ & $80193.8 \mathrm{~N} / \mathrm{mm}$ \\
Lamé constant & $\lambda$ & $110743 \mathrm{~N} / \mathrm{mm}^{2}$ & $\hat{\lambda}$ & $110743 \mathrm{~N} / \mathrm{mm}$ \\
Compression modulus & $\kappa$ & $164206.0 \mathrm{~N} / \mathrm{mm}^{2}$ & $\hat{\kappa}$ & $190936.8 \mathrm{~N} / \mathrm{mm}$ \\
Hardening coefficient & $K_{\mathrm{h}}$ & $-12.924 \mathrm{~N} / \mathrm{mm}^{2}$ & $\hat{K}_{\mathrm{h}}$ & $-12.924 \mathrm{~N} / \mathrm{mm}$ \\
Yield stress & $\sigma_{\mathrm{Y}}$ & $0 \mathrm{~N} / \mathrm{mm}^{2}$ & $\hat{\sigma}_{\mathrm{Y}}$ & $0 \mathrm{~N} / \mathrm{mm}$ \\
Initial flow stress & $\sigma_{0}$ & $450 \mathrm{~N} / \mathrm{mm}^{2}$ & $\hat{\sigma}_{0}$ & $450 \mathrm{~N} / \mathrm{mm}$ \\
Residual flow stress & $\sigma_{\infty}$ & $715 \mathrm{~N} / \mathrm{mm}^{2}$ & $\hat{\sigma}_{\infty}$ & $715 \mathrm{~N} / \mathrm{mm}$ \\
Saturation exponent & $\delta$ & 16.93 & $\hat{\delta}$ & 16.93 \\
\hline
\end{tabular}

Note that $\kappa=\lambda+2 / 3 \mu$ and $\hat{\kappa}=\hat{\lambda}+\hat{\mu}$

(a)

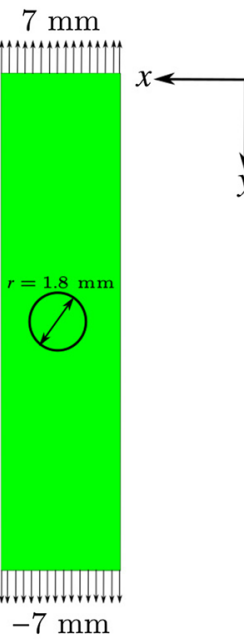

(b)

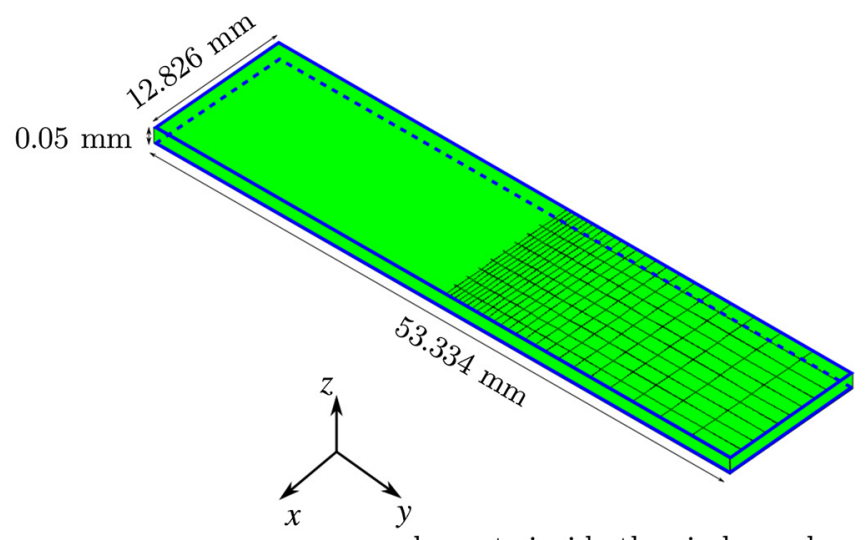

elements inside the circle weakened

top \& bottom surfaces fixed in $z$ direction

blue lines are the boundaries of energetic surfaces

Fig. 2 Strip with two elastoplastic surfaces placed at the $x-y$ surfaces. The applied boundary conditions and the finite elements are shown in a and b, respectively. The quadrature points within the circle in a are weakened to initiate the necking

\section{Numerical examples}

In this section we study the computational aspects of elastoplastic surfaces and their effects on the overall mechanical response of a body. It is important to point out that the solution procedure is robust and shows a proper rate of convergence demonstrated in "Appendix A". In the case of elasticity, we obtain exactly the quadratic rate of convergence associated with the Newton-Raphson scheme. For the purpose of demonstration the computational domain is discretized using 500 trilinear hexahedral elements. The surface inelastic response in the form of elastoplasticity is determined by the constitutive relations discussed in Sect. 3. The bulk elastoplasticity closely follows the work of [61], which for the sake of brevity is not repeated here. For the following simulations a general nonlinear saturation type hardening law of the form

$$
\begin{aligned}
& K\left(F_{\mathrm{p}}\right):=K_{\mathrm{h}} F_{\mathrm{p}}+\left[\sigma_{\infty}-\sigma_{0}\right]\left[1-\exp \left(-\delta F_{\mathrm{p}}\right)\right] \text { and } \\
& \hat{K}\left(\hat{F}_{\mathrm{p}}\right):=\hat{K}_{\mathrm{h}} \hat{F}_{\mathrm{p}}+\left[\hat{\sigma}_{\infty}-\hat{\sigma}_{0}\right]\left[1-\exp \left(-\hat{\delta} \hat{F}_{\mathrm{p}}\right)\right]
\end{aligned}
$$

in the bulk and on the surface, respectively is chosen. The corresponding material parameters for the bulk and surface together with the hardening parameters appearing in Eq. (58) are given in Table 2. Note that each pair of the material parameters in the bulk and on the surface are set to the same numerical value. By taking this measure together with the specific domain geometry shown in Fig. 2 one assures creating conditions observed in small-scale solids where surface properties are as important as those of the bulk or even dominant.

Consider now the strip shown in Fig. 2 where a constant displacement is prescribed at the two opposite faces that are $12.826 \mathrm{~mm}$ wide. The top and bottom $x-y$ surfaces are where we add the elastoplastic surfaces ${ }^{13}$ to the computational domain. The thickness of the strip (z direction) is kept constant. We point out that the reason the computational

\footnotetext{
13 Due to the negligible energetic contributions of the side surfaces only the energetics of $x-y$ surfaces are taken into account.
} 


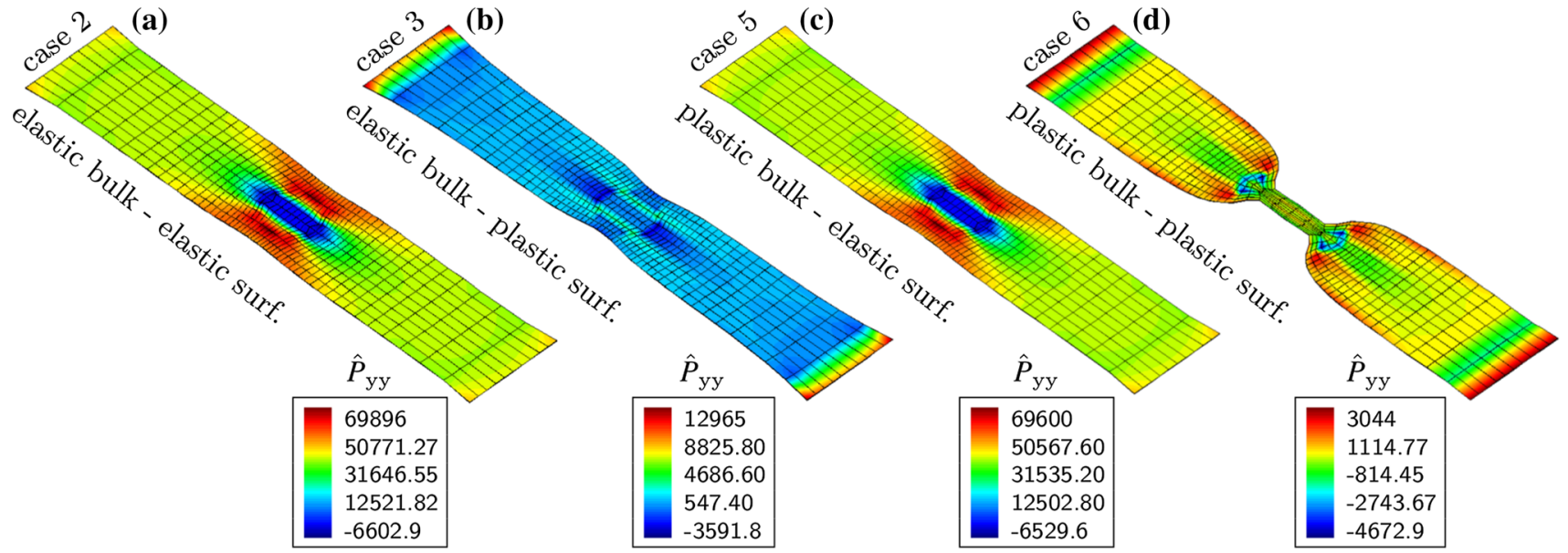

Fig. 3 Distribution of the surface Piola stress $\hat{P}_{\text {yy }} \mathrm{N} / \mathrm{mm}$

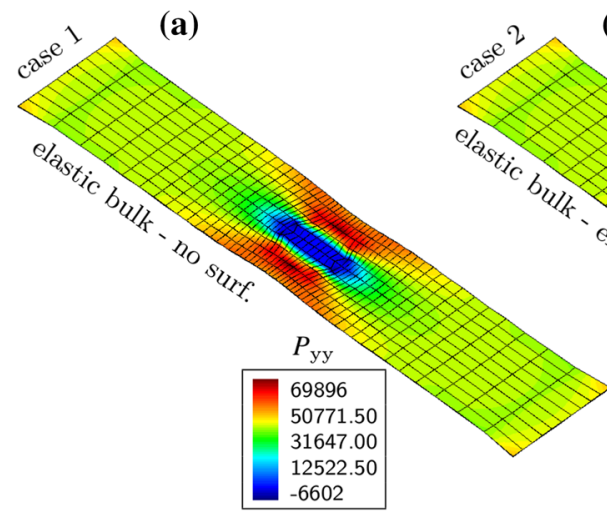

(b)

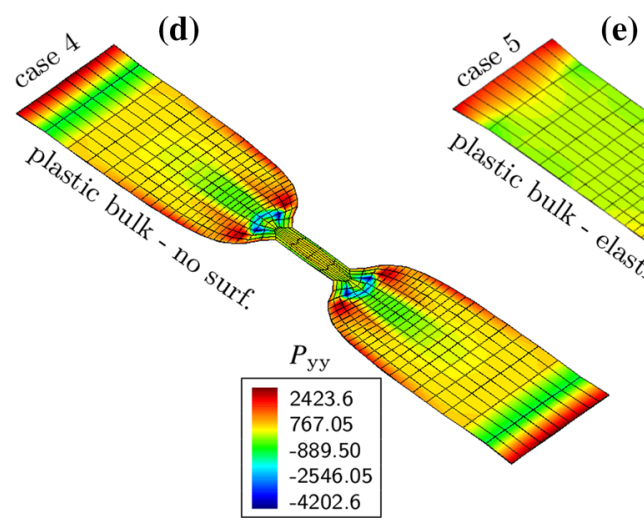

(e)

(c)

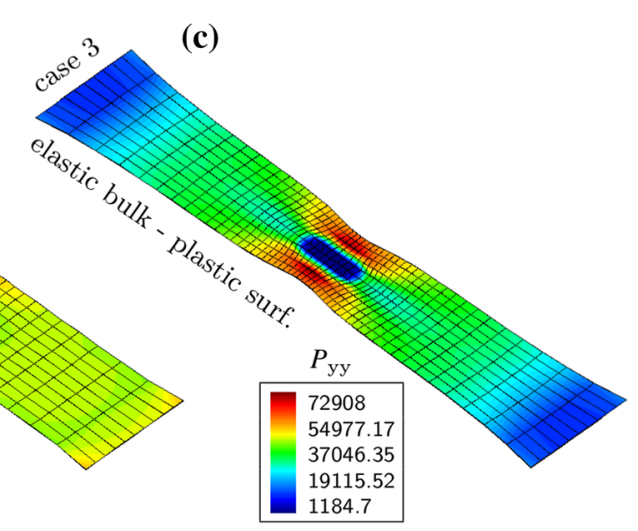

(f)

Fig. 4 Distribution of the bulk Piola stress $P_{\text {yy }} \mathrm{N} / \mathrm{mm}^{2}$

domain is chosen to be thin is to make sure that the surface area to bulk volume ratio is large enough to see the effect of surface properties on the overall mechanical behavior of the solid. To initiate the necking the area inside the circle shown in Fig. 2a is weakened. The prescribed displacement is applied in 100 equal load steps. The discretization is densified in the middle of the domain as shown in Fig. 2b. In the following examples we have devised six different cases to study surface plasticity. These cases are: case 1 elastic bulk - no surface;

case 2 elastic bulk-elastic surface; case 3 elastic bulk-plastic surface; case 4 plastic bulk - no surface; case 5 plastic bulk-elastic surface; case 6 plastic bulk-plastic surface.

Note, the utility of the current framework is to capture size effects, as a natural extension of the surface elasticity theory. 

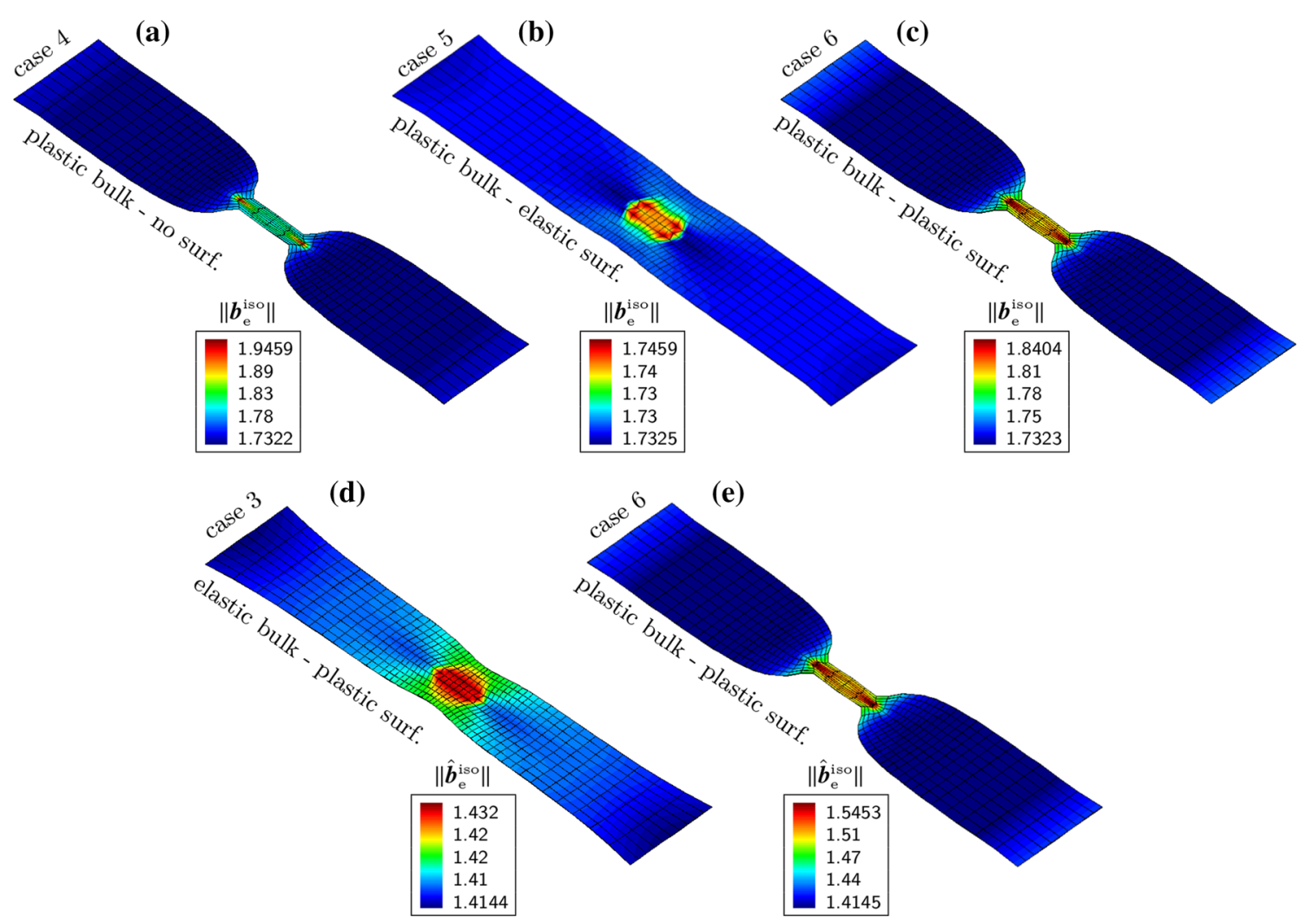

Fig. 5 Distribution of the norm of the elastic deviatoric Cauchy-Green tensor $\left\|\boldsymbol{b}_{\mathrm{e}}^{\text {iso }}\right\|$ in the bulk (a) $-(\mathbf{c})$ and $\left\|\hat{\boldsymbol{b}}_{\mathrm{e}}^{\text {iso }}\right\|$ on the surface (d), (e)

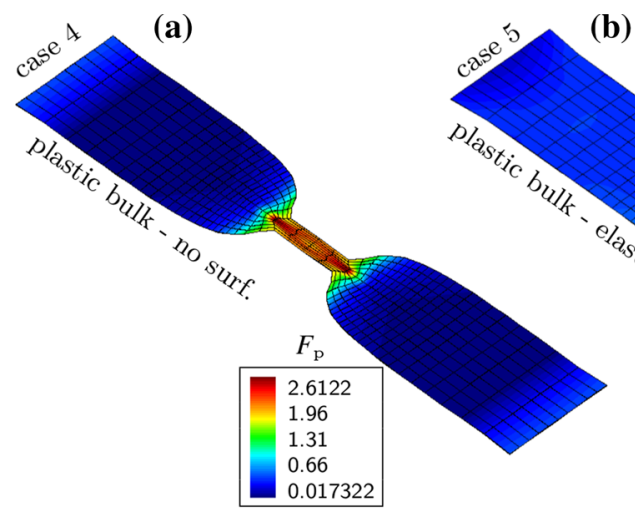

(b)
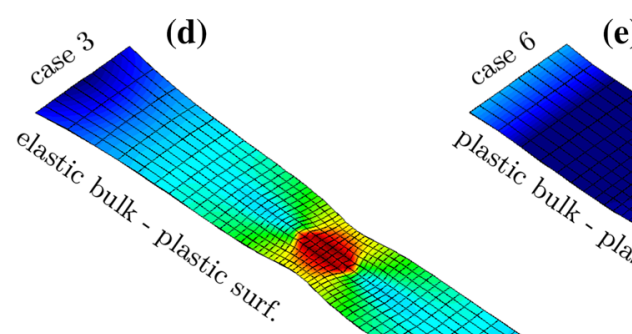

(e)
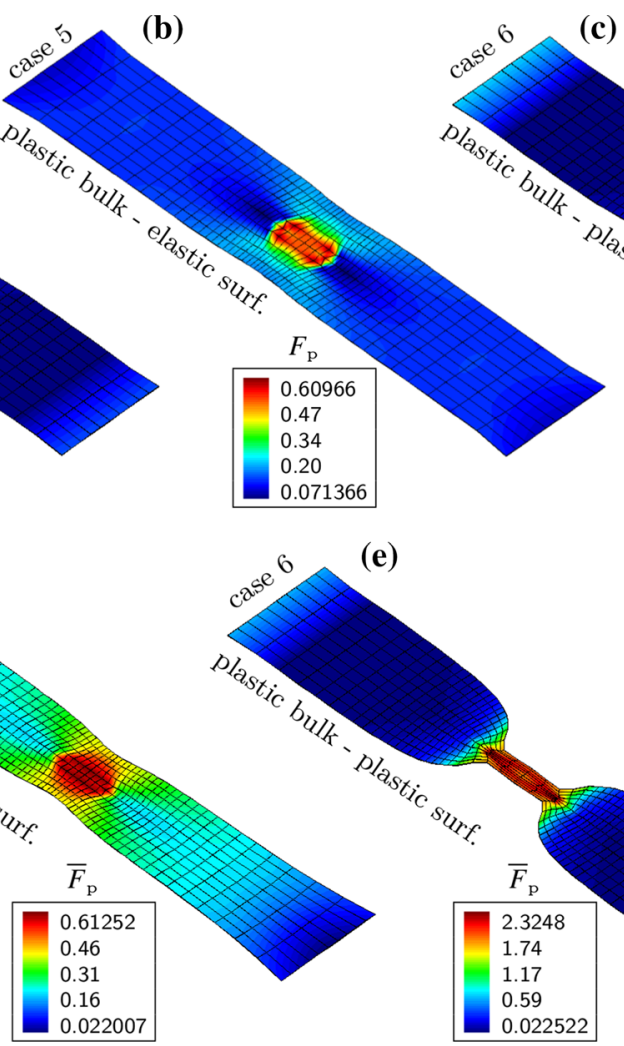

(c)
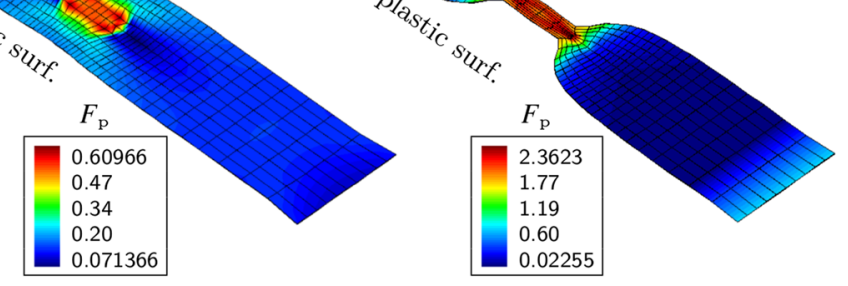


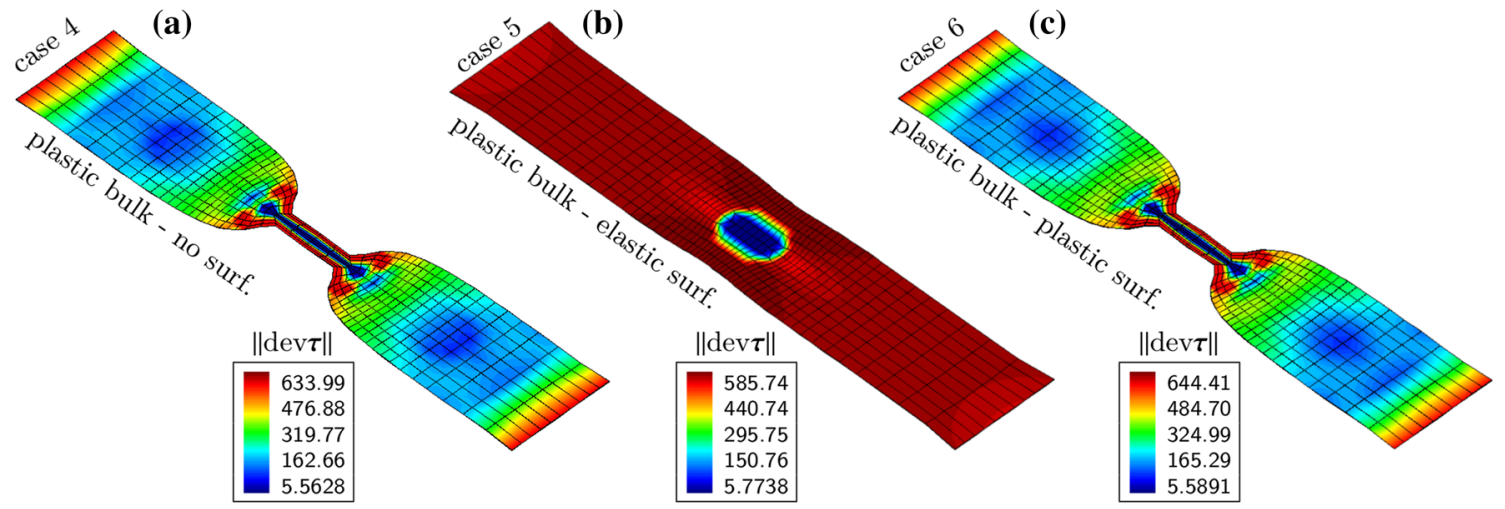

(d)

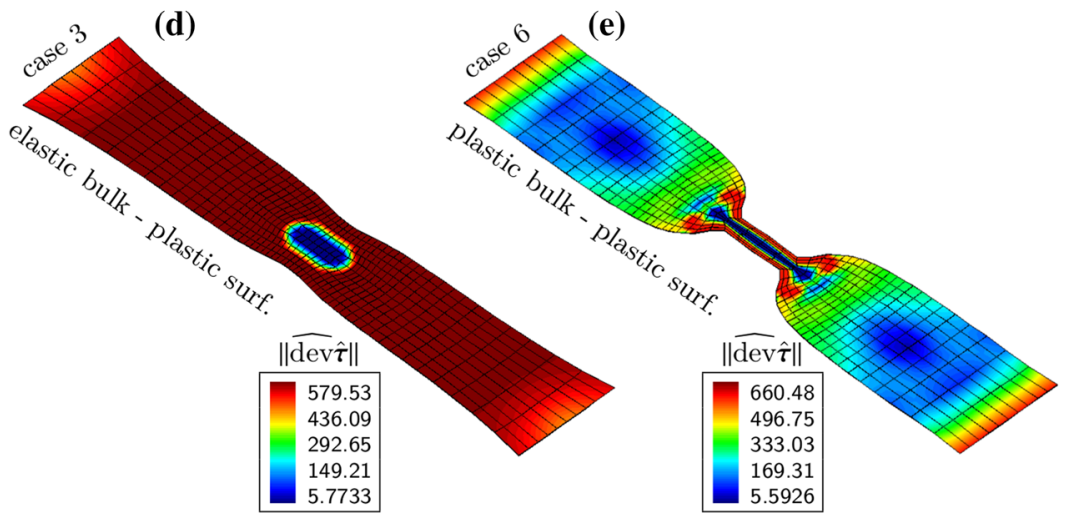

Fig. 7 Distribution of the norm of the deviatoric part of the Kirchhoff (effective von Mises) stress tensor $\|\operatorname{dev} \boldsymbol{\tau}\| \mathrm{N} / \mathrm{mm}^{2}$ in the bulk (a)-(c) and $\|\widehat{\operatorname{dev} \hat{\boldsymbol{\tau}}}\| \mathrm{N} / \mathrm{mm}$ on the surface $(\mathbf{d}),(\mathbf{e})$

However, we do not present specific examples to show the size effects here as it might be somewhat distracting for this manuscript. Nonetheless, it is obvious that the "surface/bulk" material parameters given in Table 2 are not dimensionless and one can interpret the current examples as demonstrations of size effects, as well. For instance, for a purely elastic behavior, "case 2" can be understood as "case 1" at a very small scale where the surface effects are no longer negligible. For an elasto-plastic response, "case 6" can be understood as "case 4" at a very small scale similarly.

Figure 3 depicts the surface Piola stress profile for case 2 , case 3 , case 5 and case 6 . These are the cases with elastic and elastoplastic surfaces. Due to the weakening of the quadrature points within the circle, lateral contractions are observed in Fig. 3a-c, and a necking in Fig. 3d where case 6 is considered. By allowing plastic yielding in the bulk or surface, Fig. 3b, c, a drop in the stress level is observed. Such drop is however intensified by surface plasticity. This is due to the high surface-area to bulk-volume ratio $\left(=40 \mathrm{~mm}^{-1}\right.$ in this work), which amplifies the influence of the surface behavior on the overall response of the solid. Such influence can also be seen by measuring the reaction force on the faces where the displacements are applied as drawn in Fig. 8a-c. By comparing Fig. 8a, b, it is easily understood that the force due to surface effect is 40 times higher than that due to the bulk.

In Fig. 4 the distribution of the bulk Piola stress is given. It can be seen that an elastic bulk deformation is not influenced by the presence of an elastic surface due to the assumed similar material properties of the bulk and the surface, see Fig. 4a, b. However, adding energetic surfaces to the solid increases the reaction force, compare case 1 and case 2 in Fig. 8a with those in Fig. 8c. The same observation can be made for case 4 and case 6 , an elastoplastic bulk in the absence of an energetic surface and an elastoplastic bulk with an elastoplastic surface, respectively, see Fig. 4d, f. Note that regarding case 3 , plasticity of the surface when the bulk is elastic causes more lateral contraction, see Fig. 4c, which consequently increases the stress level in the bulk. This case can be compared to its opposite, case 5 where now the bulk is plastic and the surface remains elastic. Due to more dominant surface mechanical response, less lateral contraction and thus smaller stress in the bulk are observed, see Fig. 4e.

In Figs. 5, 6 and 7 we study the important quantities with regard to the model presented in this contribution, i.e. the elastic left Cauchy-Green tensors $\left(\boldsymbol{b}_{\mathrm{e}}^{\text {iso }}, \hat{\boldsymbol{b}}_{\mathrm{e}}^{\text {iso }}\right)$, the hardening variables $\left(F_{\mathrm{p}}, \hat{F}_{\mathrm{p}}\right)$ and the deviatoric part of the Kirchhoff stresses $(\operatorname{dev} \boldsymbol{\tau}, \widehat{\operatorname{dev}} \hat{\boldsymbol{\tau}})$, in the bulk and on the surface respectively. Thus, these figures show the deformation level, the 

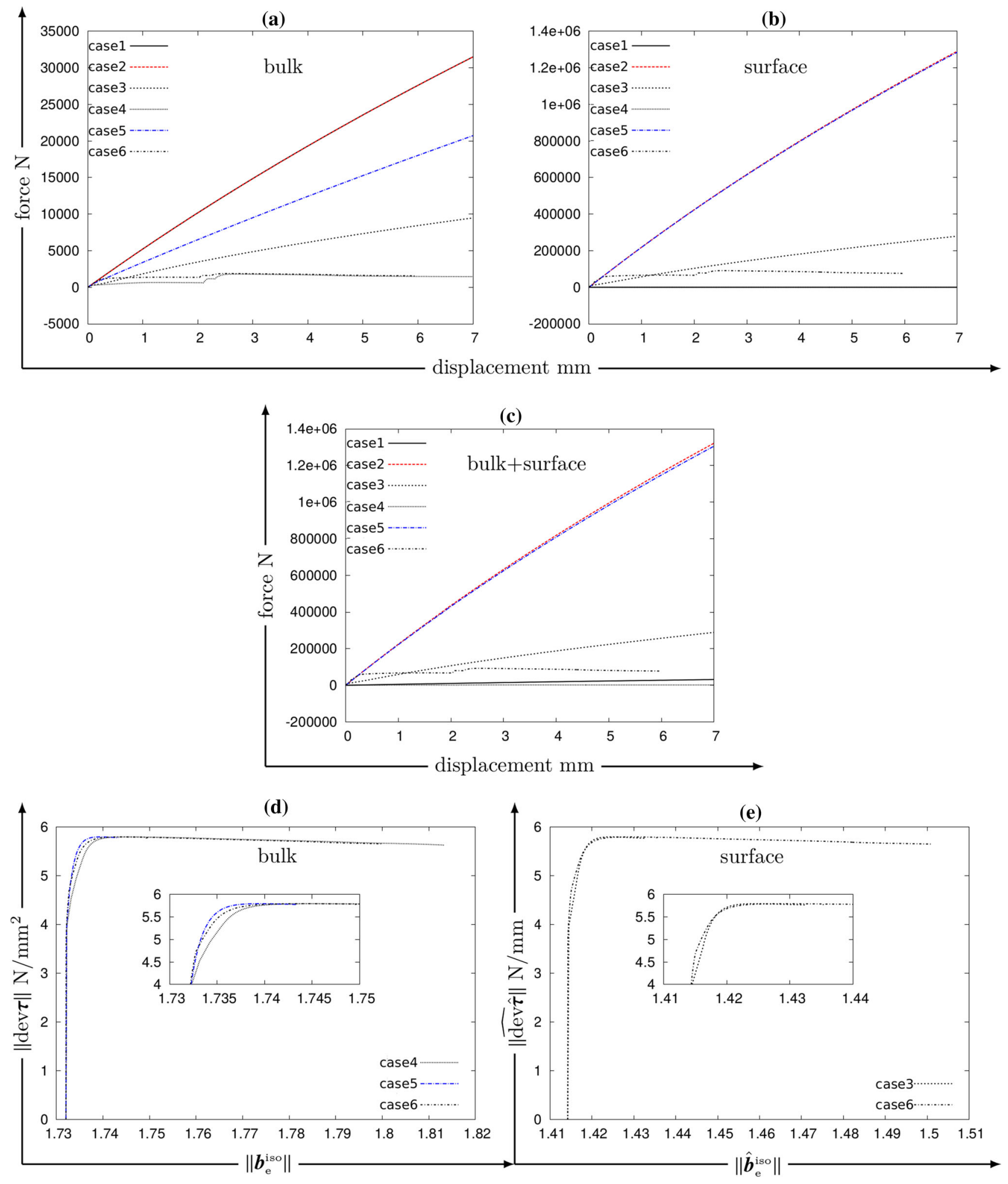

Fig. 8 Force due to the bulk (a), Force due to the surface (b) and total force (c) versus boundary displacement. Effective von Mises stress versus the norm of the elastic deviatoric Cauchy-Green tensor in the bulk (d) and on the surface (e) 
plastic yielding and von Mises effective stress of the here presented examples, respectively, for all the relevant cases, i.e case 3 -case 6 . In the first row of Fig. 5 the bulk is always elastoplastic while the surface ranges from being not present to being elastoplastic. It is clear that with an elastic surface, the elastic deviatoric deformation is constrained, see Fig. 5b. Another interesting observation is that even when the surface is allowed to be elastoplastic still a lower level of deformation is achieved as shown in Fig. 5c compared to Fig. 5a. These dissimilar levels of deformations consequently lead to different evolutions of the equivalent plastic distortion, see Fig. $6 \mathrm{a}-\mathrm{c}$, and the effective stress in the bulk, see Figs. $7 \mathrm{a}-\mathrm{c}$ and $8 \mathrm{~d}$. From Fig. $8 \mathrm{~d}$, which is drawn for one node in the middle of the domain, one can also conclude that the strongest plastic yielding in the bulk occurs for case 4 and the lowest for case 5. Figures 5, 6 and 7d, e show the elastoplastic behavior of the surface.

It is clear that when the bulk remains elastic, surface plastic deformation is constrained (see Fig. 5d compared to Fig. 5e), thus much lower values of the surface plastic equivalent distortion (see Fig. 6d compared to Fig. 6e) and von Mises effective stress are obtained (see Fig. 7d compared to Fig. 7e). We also point out that although the evolutions of the effective von Mises stresses on the surface for one node in the middle of the surface for case 5 and case 6 are almost the same (see Fig. 8e), their overall behavior is noticeably different which can be observed from the measured reaction force due to the surface, see Fig. 8b.

\section{Summary and conclusion}

A three-dimensional formulation and finite element framework for elastoplastic continua encased by elastoplastic surfaces is presented. The surfaces are endowed with their own elastoplastic constitutive behavior whereby the free energies capture the hyperelastic part of the constitutive relations. The corresponding weak forms of the balance equations including the contributions from the surfaces are given in detail. The balance equations are fully discretized in space using the finite element method. The exact consistent stiffness matrices in the bulk and on the surface are incorporated. A three-dimensional numerical example serves to elucidate the role of surface plasticity on the overall response of a body. For the sake of demonstration, we assumed that the surface response is isotropic, the plastic spin on the surface is zero and the plastic yielding of the surface is isochoric. The geometry of the computational domain is chosen so as to have a large surface area to bulk volume ratio. It is shown if the surface remains elastic, the otherwise-typical necking in the bulk is prevented and vice versa. However, lateral contraction is higher when the surface is elastoplastic and the bulk remains elastic which emphasizes the dominant role of the surface on the overall mechanical response of the solid. Necking initiates and develops to its complete form when the elastoplastic bulk is either not wrapped by an energetic surface or both the surface and the bulk are allowed to yield plastically. Although for both of the above cases the necking is fully developed and is similar in terms of deformation, their overall reaction forces are substantially different. It is seen that for the former case the force monotonically increases, whereas for the latter case the force, after an initial increase, continues to decline. The further extension of this work to non-coherent interfaces will be elaborated in a future contribution in which a traction-separation law similar to that of the cohesive zone model is assumed to relate the interface traction to the displacement jump across the interface. In addition, it is straightforward to employ more sophisticated plasticity models taking into account for instance anisotropy. Moreover, an investigation of the influences of the bulk and surface inelasticity on the thermomechanical response of a body is of great importance. Regarding the numerical implementation, one needs to also consider measures to tackle the volumetric locking and also a mesh densification study to make sure the sufficient convergence of the results. These extensions shall be discussed in later contributions.

Acknowledgements The first author gratefully acknowledges the support by the Cluster of Excellence "Engineering of Advanced Materials".

\section{Appendix A: Convergence behavior}

In this section we present some data on the convergence behavior of the computational problem at hand. The $L_{2}$ norms of the residual of few increments for some of the cases discussed in Sect. 5 are given in Table 3. 
Table $3 \quad L_{2}$ norm of the residual for case 4 , case 3 and case 6

\begin{tabular}{|c|c|c|c|c|c|c|c|c|c|}
\hline Increment & Iteration & & & & & & & & \\
\hline Case 4: pla & c bulk-no st & ace & & & & & & & \\
\hline 1 & $1.08 \mathrm{e}+3$ & $3.99 \mathrm{e}+0$ & $3.20 \mathrm{e}+0$ & $1.67 \mathrm{e}+0$ & $2.24 \mathrm{e}-02$ & $2.04 \mathrm{e}-04$ & $7.62 \mathrm{e}-06$ & $2.74 \mathrm{e}-08$ & \\
\hline 26 & $1.07 \mathrm{e}+03$ & $2.15 \mathrm{e}-01$ & $2.05 \mathrm{e}-01$ & $2.02 \mathrm{e}-02$ & $7.54 \mathrm{e}-03$ & $2.49 \mathrm{e}-03$ & $4.71 \mathrm{e}-04$ & $3.33 e-06$ & $2.17 \mathrm{e}-09$ \\
\hline 31 & $1.07 \mathrm{e}+03$ & $2.75 \mathrm{e}-01$ & $5.12 \mathrm{e}-02$ & $6.09 \mathrm{e}-04$ & $3.12 \mathrm{e}-06$ & $2.70 \mathrm{e}-08$ & & & \\
\hline 100 & $1.72 \mathrm{e}+03$ & $4.69 \mathrm{e}-01$ & $9.28 \mathrm{e}-02$ & $2.57 \mathrm{e}-02$ & $7.99 \mathrm{e}-03$ & $1.87 \mathrm{e}-03$ & $2.35 \mathrm{e}-04$ & $3.17 \mathrm{e}-06$ & $6.87 \mathrm{e}-09$ \\
\hline Case 3: ela & c bulk_-plast & surface & & & & & & & \\
\hline 1 & $4.44 e+04$ & $3.46 e+00-2$ & $8.11 \mathrm{e}-01$ & $3.68 \mathrm{e}-01$ & $1.48 \mathrm{e}-03$ & $1.22 \mathrm{e}-05$ & $8.23 \mathrm{e}-09$ & & \\
\hline 26 & $4.29 \mathrm{e}+04$ & $3.79 \mathrm{e}-02$ & $4.43 \mathrm{e}-03$ & $6.96 \mathrm{e}-07$ & $7.55 e-10$ & & & & \\
\hline 60 & $4.12 \mathrm{e}+04$ & $2.62 \mathrm{e}-02$ & $2.23 \mathrm{e}-03$ & $3.24 \mathrm{e}-05$ & $1.80 \mathrm{e}-08$ & & & & \\
\hline 100 & $4.10 \mathrm{e}+04$ & $2.51 \mathrm{e}-02$ & $2.286 \mathrm{e}-03$ & $1.06 \mathrm{e}-06$ & $3.38 \mathrm{e}-8$ & $8.36 \mathrm{e}-10$ & & & \\
\hline Case 6: pla & c bulk_plast & surface & & & & & & & \\
\hline 1 & $1.63 \mathrm{e}+04$ & $9.76 \mathrm{e}-02$ & $3.46 \mathrm{e}-03$ & $3.01 \mathrm{e}-06$ & $4.43 e-08$ & & & & \\
\hline 26 & $4.32 \mathrm{e}+04$ & $1.18 \mathrm{e}-01$ & $3.00 \mathrm{e}-02$ & $8.28 \mathrm{e}-04$ & $5.92 \mathrm{e}-06$ & $1.45 \mathrm{e}-08$ & & & \\
\hline 40 & $1.61 \mathrm{e}+04$ & $1.33 e-01$ & $1.40 \mathrm{e}-02$ & $8.66 \mathrm{e}-04$ & $6.77 \mathrm{e}-06$ & $1.82 \mathrm{e}-08$ & $3.94 \mathrm{e}-10$ & & \\
\hline 100 & $8.06 \mathrm{e}+04$ & $1.00 \mathrm{e}+00$ & $1.10 \mathrm{e}-02$ & $2.41 \mathrm{e}-04$ & $8.37 \mathrm{e}-06$ & $1.61 \mathrm{e}-09$ & & & \\
\hline
\end{tabular}

\section{References}

1. Adamson W, Gast AP(1997) Physical chemistry of surfaces. Wiley, New York

2. Bellet M (2001) Implementation of surface tension with wall adhesion effects in a three-dimensional finite element model for fluid flow. Commun Numer Methods Eng 17(8):563-579

3. Benveniste Y (2013) Models of thin interphases and the effective medium approximation in composite media with curvilinearly anisotropic coated inclusions. Int J Eng Sci 72:140-154

4. Benveniste $Y$, Miloh T (2001) Imperfect soft and stiff interfaces in two-dimensional elasticity. Mech Mater 33(6):309-323

5. Bottomley DJ, Ogino T (2001) Alternative to the Shuttleworth formulation of solid surface stress. Phys Rev B 63:165412

6. Cammarata RC (1994) Surface and interface stress effects in thin films. Prog Surf Sci 46(1):1-38

7. Cammarata RC (1997) Surface and interface stress effects on interfacial and nanostructured materials. Mater Sci Eng A 237(2):180 184

8. Chatzigeorgiou G, Javili A, Steinmann P (2013) Multiscale modelling for composites with energetic interfaces at the micro- or nanoscale. Math Mech Solids 20:1130-1145

9. Daher N, Maugin GA (1986) The method of virtual power in continuum mechanics application to media presenting singular surfaces and interfaces. Acta Mech 60(3-4):217-240

10. Davydov D, Javili A, Steinmann P (2013) On molecular statics and surface-enhanced continuum modeling of nano-structures. Comput Mater Sci 69:510-519

11. de Souza Neto EA, Peric D, Owen DRJ (2011) Computational methods for plasticity: theory and applications. Wiley, Chichester

12. dell'Isola F, Romano A (1987) On the derivation of thermomechanical balance equations for continuous systems with a nonmaterial interface. Int J Eng Sci 25:1459-1468

13. Dingreville R, Qu J, Cherkaoui M (2005) Surface free energy and its effect on the elastic behavior of nano-sized particles, wires and films. J Mech Phys Solids 53(8):1827-1854

14. Duan HL, Karihaloo BL (2007) Effective thermal conductivities of heterogeneous media containing multiple imperfectly bonded inclusions. Phys Rev B 75(6):064206
15. Duan HL, Wang J, Huang ZP, Karihaloo BL (2005a) Eshelby formalism for nano-inhomogeneities. Proc R Soc A Math Phys Eng Sci 461(2062):3335-3353

16. Duan HL, Wang J, Huang ZP, Karihaloo BL (2005b) Sizedependent effective elastic constants of solids containing nanoinhomogeneities with interface stress. J Mech Phys Solids 53(7):1574-1596

17. Duan HL, Wang J, Karihaloo BL (2009) Theory of elasticity at the nanoscale. Adv Appl Mech 42:1-68

18. Esmaeili A, Javili A, Steinmann P (2016a) A thermo-mechanical cohesive zone model accounting for mechanically energetic Kapitza interfaces. Int J Solids Struct 92-93:29-44

19. Esmaeili A, Javili A, Steinmann P (2016b) Coherent energetic interfaces accounting for in-plane degradation. Int $\mathrm{J}$ Fract 202(2):135-165

20. Esmaeili A, Javili A, Steinmann P (2016c) Highly-conductive energetic coherent interfaces subject to in-plane degradation. Math Mech Solids. https://doi.org/10.1177/1081286516642818

21. Esmaeili A, Javili A, Steinmann P (2017a) Coupled thermally general imperfect and mechanically coherent energetic interfaces subject to in-plane degradation. JoMMS 12(3):289-312

22. Esmaeili A, Steinmann P, Javili A (2017b) Non-coherent energetic interfaces accounting for degradation. Comput Mech 59(3):361383

23. Fischer FD, Simha NK, Svoboda J (2003) Kinetics of diffusional phase transformation in multicomponent elasticplastic materials. ASME J Eng Mater Technol 125:266-276

24. Fischer FD, Svoboda J (2010) Stresses in hollow nanoparticles. Int J Solids Struct 47(20):2799-2805

25. Fischer FD, Waitz T, Vollath D, Simha NK (2008) On the role of surface energy and surface stress in phase-transforming nanoparticles. Prog Mater Sci 53(3):481-527

26. Fleck NA, Willis JR (2009) A mathematical basis for straingradient plasticity theory part I: scalar plastic multiplier. J Mech Phys Solids 57(1):161-177

27. Fried E, Gurtin M (2007) Thermomechanics of the interface between a body and its environment. Contin Mech Thermodyn 19(5):253-271 
28. Fried E, Todres R (2005) Mind the gap: the shape of the free surface of a rubber-like material in proximity to a rigid contactor. J Elast 80(1-3):97-151

29. Gurtin ME (2008) A theory of grain boundaries that accounts automatically for grain misorientation and grain-boundary orientation. J Mech Phys Solids 56(2):640-662

30. Gurtin ME, Murdoch AI (1975) A continuum theory of elastic material surfaces. Arch Ration Mech Anal 57(4):291-323

31. Gutman EM (1995) On the thermodynamic definition of surface stress. J Phys Condens Matter 7(48):L663

32. Han W, Reddy BD (2013) Plasticity mathematical theory and numerical analysis. Springer, New York

33. Huang ZP, Sun L (2007) Size-dependent effective properties of a heterogeneous material with interface energy effect: from finite deformation theory to infinitesimal strain analysis. Acta Mech 190(1-4):151-163

34. Javili A, McBride A, Steinmann P (2012) Numerical modelling of thermomechanical solids with mechanically energetic (generalised) Kapitza interfaces. Comput Mater Sci 65:542-551

35. Javili A, McBride A, Steinmann P (2013b) Numerical modelling of thermomechanical solids with highly conductive energetic interfaces. Int J Numer Methods Eng 93(5):551-574

36. Javili A, McBride A, Steinmann P (2013c) Thermomechanics of solids with lower-dimensional energetics: on the importance of surface, interface, and curve structures at the nanoscale. A unifying review. Appl Mech Rev 65(1):010802

37. Javili A, Steinmann P (2009) A finite element framework for continua with boundary energies. Part I: the two-dimensional case. Comput Methods Appl Mech Eng 198(27-29):2198-2208

38. Javili A, Steinmann P (2010a) A finite element framework for continua with boundary energies. Part II: the three-dimensional case. Comput Methods Appl Mech Eng 199(9-12):755-765

39. Javili A, Steinmann P (2010b) On thermomechanical solids with boundary structures. Int J Solids Struct 47(24):3245-3253

40. Johnson WC (2000) Superficial stress and strain at coherent interfaces. Acta Mater 48:433-444

41. Kaptay G (2005) Classification and general derivation of interfacial forces, acting on phases, situated in the bulk, or at the interface of other phases. J Mater Sci 40:2125-2131

42. Kramer D, Weissmüller J (2007) A note on surface stress and surface tension and their interrelation via Shuttleworths equation and the Lippmann equation. Surf Sci 601(14):3042-3051

43. Leo PH, Sekerka RF (1999) The effect of surface stress on crystalmelt and crystal-crystal equilibrium. Springer, Berlin, pp 176-195

44. Levitas VI, Javanbakht M (2010) Surface tension and energy in multivariant martensitic transformations: phase-field theory, simulations, and model of coherent interface. Phys Rev Lett 105(16): 165701

45. Miller RE, Shenoy VB (2000) Size-dependent elastic properties of nanosized structural elements. Nanotechnology 11:139-147

46. Moeckel GP (1975) Thermodynamics of an interface. Arch Ration Mech Anal 57(3):255-280

47. Müller P, Saúl A (2004) Elastic effects on surface physics. Surf Sci Rep 54(5):157-258

48. Navti SE, Ravindran K, Taylor C, Lewis R (1997) Finite element modelling of surface tension effects using a Lagrangian-Eulerian kinematic description. Comput Methods Appl Mech Eng 147:4160

49. Park HS, Klein PA (2007) Surface Cauchy-Born analysis of surface stress effects on metallic nanowires. Phys Rev B 75:085408
50. Rusanov AI (1996) Thermodynamics of solid surfaces. Surf Sci Rep 23:173-247

51. Saksono PH, Perić D (2006) On finite element modelling of surface tension: variational formulation and applications-part I: quasistatic problems. Comput Mech 38(3):265-281

52. Saksono PH, Perić D (2006) On finite element modelling of surface tension: variational formulation and applications-part II: dynamic problems. Comput Mech 38(3):251-263

53. Sharma P, Ganti S (2004) Size-dependent Eshelbys tensor for embedded nano-inclusions incorporating surface/interface energies. J Appl Mech 71(5):663-671

54. Sharma P, Ganti S, Bhate N (2003) Effect of surfaces on the sizedependent elastic state of nano-inhomogeneities. Appl Phys Lett 82(4):535-537

55. Sharma P, Wheeler LT (2007) Size-dependent elastic state of ellipsoidal nano-inclusions incorporating surface/interface tension. J Appl Mech 74(3):447-454

56. She H, Wang B (2009) A geometrically nonlinear finite element model of nanomaterials with consideration of surface effect. Finite Elem Anal Des 45:463-467

57. Simha NK, Bhattacharya K (1997) Equilibrium conditions at corners and edges of an interface in a multiphase solid. Mater Sci Eng A238:32-41

58. Simha NK, Bhattacharya K (1998) Kinetics of phase boundaries with edges and junctions. J Mech Phys Solid 46:2323-2359

59. Simo JC (1988a) A framework for finite strain elastoplasticity based on maximum plastic dissipation and the multiplicative decomposition. part I: continuum formulation. Comput Methods Appl Mech Eng 66(2):199-219

60. Simo JC (1988b) A framework for finite strain elastoplasticity based on maximum plastic dissipation and the multiplicative decomposition. Part II: computational aspects. Comput Methods Appl Mech Eng 68(1):1-31

61. Simo JC, Hughes TJR (1998) Computational inelasticity. Springer, New York

62. Simo JC, Meschke G (1993) A new class of algorithms for classical plasticity extended to finite strains. Application to geomaterials. Comput Mech 11(4):253-278

63. Steigmann DJ, Ogden RW (1999) Elastic surface-substrate interactions. Proc R Soc Lond A Math Phys Eng Sci 455(1982):437-474

64. Steinmann P (2008) On boundary potential energies in deformational and configurational mechanics. J Mech Phys Solids 56(3):772-800

65. Steinmann P, Häsner O (2005) On material interfaces in thermomechanical solids. Arch Appl Mech 75(1):31-41

66. Wei G, Shouwen Y, Ganyun H (2006) Finite element characterization of the sizedependent mechanical behaviour in nanosystems. Nanotechnology 17:1118-1122

67. Yang F (2006) Effect of interfacial stresses on the elastic behavior of nanocomposite materials. J Appl Phys 99:054306

68. Yvonnet J, Mitrushchenkov A, Chambaud G, He Q-C (2011) Finite element model of ionic nanowires with size-dependent mechanical properties determined by ab initio calculations. Comput Methods Appl Mech Eng 200(5-8):614-625

69. Yvonnet J, Quang HL, He QC (2008) An XFEM/level set approach to modelling surface/interface effects and to computing the sizedependent effective properties of nanocomposites. Comput Mech 42:119-131 